\title{
Logarithmic Corrections to Rotating Extremal Black Hole Entropy in Four and Five Dimensions
}

\author{
Ashoke Sen \\ Harish-Chandra Research Institute \\ Chhatnag Road, Jhusi, Allahabad 211019, India \\ E-mail: sen@mri.ernet.in
}

\begin{abstract}
We compute logarithmic corrections to the entropy of rotating extremal black holes using quantum entropy function i.e. Euclidean quantum gravity approach. Our analysis includes five dimensional supersymmetric BMPV black holes in type IIB string theory on $T^{5}$ and $K 3 \times S^{1}$ as well as in the five dimensional CHL models, and also non-supersymmetric extremal Kerr black hole and slowly rotating extremal Kerr-Newmann black holes in four dimensions. For BMPV black holes our results are in perfect agreement with the microscopic results derived from string theory. In particular we reproduce correctly the dependence of the logarithmic corrections on the number of $\mathrm{U}(1)$ gauge fields in the theory, and on the angular momentum carried by the black hole in different scaling limits. We also explain the shortcomings of the Cardy limit in explaining the logarithmic corrections in the limit in which the (super)gravity description of these black holes becomes a valid approximation. For non-supersymmetric extremal black holes, e.g. for the extremal Kerr black hole in four dimensions, our result provides a stringent testing ground for any microscopic explanation of the black hole entropy, e.g. Kerr/CFT correspondence.
\end{abstract}




\section{Contents}

1 Introduction and summary $\quad 2$

2 General strategy $\quad 9$

2.1 Partition function in the near horizon geometry . . . . . . . . . . . . 9

2.2 Logarithmic correction from the non-zero modes . . . . . . . . . . . . 11

2.3 Zero mode contribution . . . . . . . . . . . . . . . . . . 13

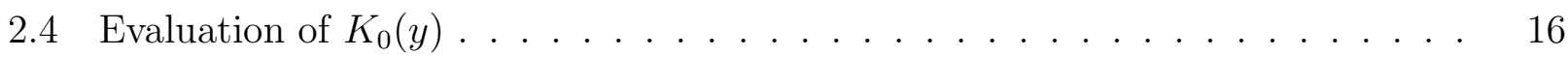

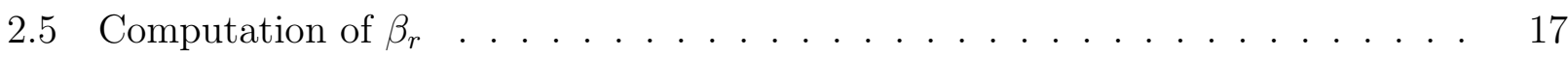

2.6 Computation of $\int d^{d} y G(y) \bar{K}^{r}(y) \ldots \ldots \ldots \ldots$

\begin{tabular}{ll|l}
3 & Extremal Kerr black hole in four dimensions & 19 \\
\hline
\end{tabular}

4 BMPV black holes $\quad 22$

4.1 The near horizon geometry . . . . . . . . . . . . . . . . 22

4.2 Ensemble choice and index from entropy . . . . . . . . . . . . . . 25

4.3 Logarithmic corrections . . . . . . . . . . . . . . . . . 2 27

5 Microscopic analysis of the BMPV black hole entropy $\quad 28$

5.1 Expression for the index in type IIB on $K 3 \times S^{1} / \mathbb{Z}_{\mathbf{N}} \ldots \ldots . . . . . . . .28$

5.2 Evaluation of the index . . . . . . . . . . . . . . . . . 30

5.3 Removal of the additional hair contribution . . . . . . . . . . . . . . 34

5.4 BMPV black hole in type IIB on $T^{5} \ldots$. . . . . . . . . . . . . . . . . 35

5.5 Comparison with the Cardy limit . . . . . . . . . . . . . . 37

6 Slowly rotating black holes $\quad 38$

6.1 Slowly rotating BMPV black holes . . . . . . . . . . . . . . 38

6.2 Slowly rotating extremal Kerr-Newmann black hole in four dimensions . . . . 39

A Counting of zero modes in $A d S_{2}$

\section{Introduction and summary}

Supersymmetric extremal black holes enjoy a certain set of non-renormalization properties, and this makes them a very useful testing ground for comparing the macroscopic predictions 
for the entropy against the microscopic entropy [1,2]. In particular for a class of $\mathcal{N}=4$ supersymmetric string theories in four dimensions one can use Wald's formula [3 6] adapted to BPS black holes [7 9] for computing higher derivative corrections to the black hole entropy, and the results are in remarkable agreement with the microscopic results [10 20]. Nevertheless, as there have been other attempts to explain extremal black hole entropy without making direct use of string theory [21,22], it is useful to explore to what extent string theory can do better than these general methods, particularly in situations where quantum gravity corrections to black hole entropy become important.

A generalization of Wald's formula, based on path integral over various fields in the near horizon geometry of the black hole, can be used to compute quantum corrections to the extremal black hole entropy [23, 24].1. Recently this method has been used to compute logarithmic corrections to the entropy in a class of four dimensional $\mathcal{N} \geq 2$ supersymmetric string theories [25 28]. These corrections are particularly interesting since unlike higher derivative corrections which are highly sensitive to the specific string theory under consideration and may not exist e.g. in a different approach to quantizing gravity, the logarithmic corrections exist in any generic theory of gravity. Furthermore they are determined purely from the low energy data - spectrum of massless fields and their interactions - and are insensitive to the spectrum of massive fields and higher derivative corrections. At the same time, they are not universal since they are sensitive to what kind of massless fields the theory has, and also their interactions. Thus once we compute these logarithmic corrections from the low energy data, they will provide a testing ground for any proposed ultraviolet completion of gravity and the description of black hole microstates in this theory. A microscopic theory that does not reproduce the correct logarithmic corrections must not be the correct microscopic theory of gravity.

So far the computations have been done for spherically symmetric black hole solutions and whenever microscopic results are available in string theory, e.g. in $\mathcal{N}=4$ and $\mathcal{N}=8$ string theories in four dimensions [10,20,29], the microscopic and the macroscopic results for the logarithmic corrections are in perfect agreement. For $\mathcal{N}=2,3$ and 6 supersymmetric theories in four dimensions there are definite predictions for the logarithmic corrections from

\footnotetext{
${ }^{1}$ As has already been emphasized in the past, extremal black holes refer to extremal limit of non-extremal black holes. On the macroscopic side this is apparent from the form of the Euclidean $A d S_{2}$ metric (2.1), which is an analytic continuation of the Lorentzian near horizon metric $-\left(r^{2}-1\right) d t^{2}+d r^{2} /\left(r^{2}-1\right)(r=\cosh \eta$, $t=-i \theta)$. This near horizon geometry arises in the zero temperature limit of black holes and still has a bifurcate Killing horizon at $r=1$. The gravity / string theory partition function in this geometry is then compared with the microscopic partition function $\operatorname{Tr}\left(e^{-H / T}\right)$ in the zero temperature limit, which, for a gapped system, approaches $d_{0} e^{-E_{0} / T}$ where $E_{0}$ is the ground state energy and $d_{0}$ is the ground state degeneracy.
} 


\begin{tabular}{|c|l|}
\hline Scaling & logarithmic correction to the entropy \\
\hline \hline$Q_{1}, Q_{5}, n \sim \Lambda, J \sim \Lambda^{3 / 2}$ & $-\frac{1}{4}\left(n_{V}-3\right) \ln \Lambda$ \\
\hline$Q_{1}, Q_{5}, n \sim \Lambda, J=0$ & $-\frac{1}{4}\left(n_{V}+3\right) \ln \Lambda$ \\
\hline \hline
\end{tabular}

Table 1: Logarithmic corrections to the entropy of a BMPV black hole in type IIB string theory compactified on $K 3 \times S^{1} / \mathbb{Z}_{\mathbf{N}}$ for $\mathbf{N}=1,2,3,5,7 . n_{V} \equiv \frac{48}{\mathbf{N}+1}+3$ denotes the total number of $U(1)$ gauge fields in the five dimensional theory. The entropy given in this table counts the number of states with fixed values of the total angular momentum as well as the third component of the angular momentum and hence does not include the degeneracy factor of $(J+1)$ from the multiplicity of $S U(2)_{L}$ spin $J / 2$ states. Each of the results in this table has been calculated independently in the macroscopic and microscopic description and in each case we find perfect agreement between the two sides.

the macroscopic side [27, 28], but no microscopic results are available yet. Some results for non-BPS black holes are also available [27] from the macroscopic side and yet others have been proposed [28] but there are no known microscopic results to compare them with.

In this paper we extend the computation to extremal rotating black holes. Before we describe our results it is necessary to say a few words about the ensemble in which we compute the entropy. It follows from the general analysis of [23] that the extremal black hole computes the entropy in the microcanonical ensemble, carrying fixed charges associated with all the gauge fields on $A d S_{2}$. In this case the massless gauge fields on $A d S_{2}$ include all the Maxwell fields of the original theory as well as the gauge fields arising out of the metric due to rotational isometries of the black hole solution. For a rotating black hole carrying $\mathrm{SU}(2)$ angular momentum $J_{3}=j$, only the isometry associated with rotation about the third axis gives rise to massless gauge fields in $A d S_{2}$ and hence the entropy computed by quantum entropy function counts states with fixed $J_{3}=j$ without any restriction on the total angular momentum. We shall denote by $\widetilde{d}(j)$ the degeneracy of states with this restriction. We can also introduce another ensemble in which we fix the total angular momentum $\vec{J}^{2}$ to $j(j+1)$ besides fixing $J_{3}$ to $j$. The corresponding degeneracy will be denoted by $d(j)$. The latter is the relevant number for $j=0$ since a black hole with $j=0$ will have full $S U(2)$ isometry and as a consequence quantum entropy function will count states for which all components of the angular momentum are fixed to 0 . We note however that $d(j)$ and $\widetilde{d}(j)$ are related by the simple relation $d(j)=\widetilde{d}(j+1)-\widetilde{d}(j)$; thus knowledge of one determines the other. We shall in fact see that 
for generic $j, \widetilde{d}(j+1)$ is related to $\widetilde{d}(j)$ by a multiplicative factor of order unity (it follows from the fact that entropy scales in the same way as $j)$, and hence $\ln d(j)$ and $\ln \widetilde{d}(j)$ differ by an additive term of order unity and have identical logarithmic corrections. The situation changes when $j$ takes value that is parametrically smaller than the generic value; this case will be discussed separately later.

Another remark that is relevant for the supersymmetric black holes is the relation between index and entropy. Black holes compute degeneracies whereas the quantity that is robust against changes of parameters and hence can be compared between the macroscopic and the microscopic results is an appropriate supersymmetric index. It has however been argued in [30,31] that for a supersymmetric black hole quantum entropy function also computes an index and hence we can directly compare the results obtained from quantum entropy function with the index computed in the microscopic theory. This argument will be reviewed in $\$ 4.2$.

We consider two classes of examples, - BMPV black holes in five dimensions [2] and extremal rotating black holes in four dimensions. For the former explicit microscopic results are known [18,32] and our results are in perfect agreement with these results. We have shown in table 1 the logarithmic corrections to the entropy of a BMPV black hole in type IIB string theory compactified on $K 3 \times S^{1} / \mathbb{Z}_{\mathbf{N}}$ where $\mathbf{N}$ is a prime integer $(1,2,3,5$ or 7$)$, and the $\mathbb{Z}_{\mathbf{N}}$ acts as a shift along $S^{1}$ and a transformation on $K 3$ that preserves 16 supersymmetries. For $\mathbf{N}=1$ this reduces to the original BMPV black hole in type IIB in $K 3 \times S^{1}$ [2] while other values of $\mathbf{N}$ correspond to BMPV black holes in five dimensional CHL models [33 36]. $n_{V} \equiv \frac{48}{\mathbf{N}+1}+3$ denotes the total number of $U(1)$ gauge fields in the five dimensional theory including any vector field that can come from dualizing a 2-form field, e.g. for $\mathbf{N}=1$ we have $n_{V}=27$. The classical black hole solution under consideration carries $Q_{1}$ units of D1-brane charge along $S^{1}, Q_{5}$ units of D5-brane charge along $K 3 \times S^{1},-n / \mathbf{N}$ units of momentum along $S^{1}$ and $S O(4)=S U(2)_{L} \times S U(2)_{R}$ angular momentum $\vec{J}_{R}^{2}=0, J_{1 L}=J_{2 L}=0, J_{3 L}=\frac{J}{2}$. The table shows the results for the logarithmic corrections to the entropy in the limit when $\Lambda$ is large. Each of the results in this table has been calculated independently in the macroscopic and microscopic description and in each case we find perfect agreement between the two sides. For this comparison we need to ensure the correct choice of ensemble on the microscopic side, so that the result can be compared with the microcanonical entropy that the macroscopic side computes. There are two relevant ensembles: in the first one $\vec{J}_{R}^{2}=0$ and $J_{3 L}$ is fixed at $J / 2$ and in the second one $\vec{J}_{R}^{2}=0, J_{3 L}$ is fixed at $J / 2$ and $\vec{J}_{L}^{2}$ is fixed at $\frac{J}{2}\left(\frac{J}{2}+1\right)$. Both ensembles of course have all the charges fixed. As discussed above, a direct macroscopic calculation gives the 
entropy in the first ensemble for $J \neq 0$ and in the second ensemble for $J=0$. We have however used the known relation between the entropies in the two ensembles to express all the results in table 1 in the second ensemble. Also important for this comparison is the relation between the index and entropy for black holes preserving four supersymmetries [30,31] so that we can sensibly compare the entropy on the macroscopic side to the logarithm of the index computed on the microscopic side. Finally, this analysis can be easily generalized to other allowed values of $\mathbf{N}$ and with K3 replaced by $T^{4}$ using the results of [19,20], with the only difference that the simple relation between $\mathbf{N}$ and $n_{V}$ will be lost, and some of the modular functions which will appear later in our microscopic formula will have a more complicated form.

Our results also hold for BMPV black holes in type IIB string theory compactified on $T^{5}$. In fact on the macroscopic side there is no difference between the analysis in the CHL models or $T^{5}$ compactifications, except that in the latter case $n_{V}$ has a specific value 27 . Thus for the scaling $Q_{1}, Q_{5}, n \sim \Lambda, J \sim \Lambda^{3 / 2}$ the result for logarithmic correction to the entropy takes the form

$$
-6 \ln \Lambda
$$

On the other hand for $Q_{1}, Q_{5}, n \sim \Lambda, J=0$ we get a logarithmic correction of the form:

$$
-\frac{15}{2} \ln \Lambda
$$

The computation on the microscopic side is quite different since we have a completely different microscopic formula [37]. Nevertheless the final results agree precisely with (1.1) and (1.2).

In computing the entropy of the black hole from the microscopic side one often invokes the Cardy formula [38, 39]. Since the underlying CFT has central charge of order $Q_{1} Q_{5}$ and the black hole describes an ensemble of states with vanishing $\bar{L}_{0}$ eigenvalue and $L_{0}$ eigenvalue of order $n$, the central charge scales as $\Lambda^{2}$ and the $L_{0}$ eigenvalue scales as $\Lambda$ in the scaling limit we are considering. Thus the Cardy formula is not directly applicable. But one often uses the intuition that the CFT describing the D1-D5 system is a sigma model whose target space is the symmetric product of $Q_{1} Q_{5}$ copies of $K 3$ (or $T^{4}$ ) [40], and the twisted sector of this theory has long string excitations whose dynamics is described by an effective CFT with central charge of order one and $L_{0}$ eigenvalue of order $Q_{1} Q_{5} n \sim \Lambda^{3}$ [41,42]. This effectively amounts to keeping $Q_{1}, Q_{5}$ fixed and scaling $n$ as $\Lambda^{3}$, and Cardy formula can be applied. We have examined the microscopic formula to examine the behaviour of the index in this limit with $J \sim \Lambda^{3 / 2-\alpha}$ for $\alpha \geq 0$. We find that while for type IIB string theory on $T^{5}$ the result agrees with the one shown in table 1 for $n_{V}=27$, for the CHL models (including type IIB string theory on $K 3 \times S^{1}$ ) the 
logarithmic correction to the $\log$ of the index is given by $-\alpha \ln \Lambda$, which is quite different from the actual result $-\frac{1}{4}\left(n_{V}-3+4 \alpha\right) \ln \Lambda$ (see table 1 for $\alpha=0$ and eq. (1.4) below for general $\alpha$ ). This mismatch for the CHL models shows that it is not always possible to extract the logarithmic correction to black hole entropy by examining the corresponding formula in the Cardy limit.

For non-supersymmetric extreme Kerr black holes in four dimensions our result for logarithmic correction to the entropy takes the form:

$$
\frac{1}{180} \ln A_{H}\left(2 n_{S}-26 n_{V}+7 n_{F}-\frac{233}{2} n_{3 / 2}+64\right),
$$

if, besides gravity, the theory contains $n_{S}$ minimally coupled massless scalar, $n_{V}$ minimally coupled massless U(1) gauge fields, $n_{F}$ minimally coupled massless Dirac fermions and $n_{3 / 2}$ minimally coupled massless real Rarita-Schwinger fields. Here $A_{H}$ is the area of the event horizon. It will be interesting to explore if (1.3) can be explained using Kerr/CFT correspondence [22], which is an attempt to explain the origin of extremel Kerr black hole entropy by postulating the existence of a $(1+1)$ dimensional conformal field theory dual to gravity in the near horizon geometry of the black hole.

Besides the scaling limits discussed above, we also consider the cases of slowly rotating black holes - black holes for which the angular momentum is parametrically smaller than the other charges so that the contribution of the angular momentum to the entropy becomes negligible in the scaling limit. For example for BMPV black holes we can consider the scaling limit $Q_{1}, Q_{5}, n \sim \Lambda$ and $J \sim \Lambda^{\frac{3}{2}-\alpha}$ for some positive constant $\alpha$. In this case the computation of the macroscopic entropy requires some additional assumptions about the spectrum of the kinetic operator in the background of such slowly rotating black holes. With this our result for the logarithmic correction to the entropy, in the ensemble in which $\vec{J}_{R}^{2}=0, J_{3 L}$ is fixed to be $J / 2$ and $\vec{J}_{L}^{2}$ is fixed to be $\frac{J}{2}\left(\frac{J}{2}+1\right)$, is given by

$$
-\frac{1}{4}\left(n_{V}-3+4 \alpha\right) \ln \Lambda \text {. }
$$

This agrees with the microscopic results in the same ensemble. We also consider slowly rotating extremal Kerr-Newmann black hole carrying charge $q$ and angular momentum $J$, with rotation parameter $\gamma \equiv J / q^{2} \sim J / A_{H}$ small. In this case we get a logarithmic correction of the form

$$
-\frac{1}{180}\left(784+n_{S}+62 n_{V}+11 n_{F}\right) \ln A_{H}+\ln \gamma,
$$


if the theory contains, besides the metric and the Maxwell field under which the black hole is charged, $n_{S}$ minimally coupled massess scalar field, $n_{V}$ minimally coupled additional massless vector fields and $n_{F}$ minimally coupled massless Dirac fermions. (1.5) refers to the entropy computed in an ensemble with fixed charges, $J_{3}=J$ and $\vec{J}^{2}=J(J+1)$.

Various other earlier approaches to computing logarithmic corrections to black hole entropy can be found in [39,43, 58]. Since in [27] we have given a detailed comparison between our method and the method of [47,58, 59] - which is closest to the method we use - we shall not repeat the discussion here. However it will be prudent to point out that the naive application of the method of [47,58,59] would give zero result for the logarithmic correction to the BMPV black hole entropy, since the trace anomaly on which their computation is based vanishes in odd dimensions. The reason that we get a non-zero result is due to the zero modes which must be treated separately, and is not correctly accounted for in the trace anomaly based approach. For extremal Kerr black hole the analysis of [47,58,59] would correctly give the dependence on $n_{S}, n_{V}, n_{F}$ and $n_{3 / 2}$ in (1.3) but would give the constant term to be 424 instead of 64 . This is again due to the additional corrections due to graviton zero modes which have been included in (1.3).

The rest of the paper is organized as follows. In \$2 we describe the procedure for computing logarithmic correction to the entropy of an extremal rotating black hole, generalizing our earlier analysis for spherically symmetric extremal black holes. In $\$ 3$ we apply the results of $\$ 2$ to compute the logarithmic correction to the entropy of an extremal Kerr black hole in four dimensions, arriving at the result (1.3). In $\$ 4$ we repeat the analysis for five dimensional BMPV black hole in the limit when all the charges are scaled simultaneously by some large number $\Lambda$, and arrive at the results given in table 1. In $\$ 5$ we carry out the microscopic analysis of the entropy of BMPV black holes in the same scaling limits and reproduce the results of table 1 and eq.(1.4). We find however that while the macroscopic analysis takes identical form for toroidal compactification of type IIB string theory and the CHL models, the microscopic analysis for these models are quite different. Nevertheless at the end the microscopic and the macroscopic results agree for all models. In $\$ 6$ we compute logarithmic correction to the entropy of slowly rotating BMPV black holes in five dimensions and extremal Kerr-Newmann black hole in four dimensions and arrive at eqs.(1.4) and (1.5). In appendix $\AA$ we review the counting of zero modes for different fields which play an important role in the macroscopic computation of logarithmic corrections to the entropy.

We shall end this section by drawing some general lessons from our analysis. Since quantum 
entropy function approach is rooted in Euclidean quantum gravity [60, what the results of this paper and those in [25, 26] show is that Euclidean quantum gravity provides us with an accurate description of the quantum corrections to black hole entropy. Of course Euclidean quantum gravity path integral suffers from the usual ultraviolet divergences of gravity, but some computations, e.g. logarithmic corrections to black hole entropy, are insensitive to the ultraviolet completion of the theory and are determined solely by the infrared theory [59]. Thus we can in principle use Euclidean quantum gravity to calculate even the entropy of nonextremal black holes, and any consistent ultraviolet completion of the theory must reproduce these results. This could provide a strong check on any proposed ultraviolet completion of gravity, if the latter provides an independent derivation of the logarithmic correction to the black hole entropy.

\section{General strategy}

In [25 27] we used the quantum entropy function [23] to compute logarithmic corrections to the entropy of four dimensional spherically symmetric extremal black holes with $A d S_{2} \times S^{2}$ near horizon geometry. In this section we shall generalize this analysis to rotating extremal black holes in arbitrary dimensions.

\subsection{Partition function in the near horizon geometry}

Rotating extremal black holes are expected to have an $A d S_{2}$ factor in their near horizon geometry [61 65], possibly fibered over some other directions. Besides the $A d S_{2}$ factor the near horizon geometry consists of various other compact directions, some of which could be inherited from the compact directions of the asymptotic geometry while others could be the angular coordinates of the non-compact part of the asymptotic space-time. Our goal will be to compute logarithmic corrections to the entropy in certain scaling limit in which the various charges grow in a certain way. In this limit some of these compact directions grow in size while the others remain fixed in size. We shall label by $K$ the space spanned by the compact directions which grow in size, irrespective of whether they are inherited from the compact coordinates or the angular coordinates of the asymptotic space-time. Let $d$ be the dimension of $K$. We shall Fourier decompose all string fields along the compact directions of fixed size and treat this as a $(d+2)$ dimensional theory with the $(d+2)$ coordinates containing the coordinates of $A d S_{2}$ and $K$. In what follows we shall denote the coordinates of $A d S_{2}$ by $x$, the 
coordinates along $K$ by $y$ and the coordinates $(x, y)$ collectively by $z$. We shall for simplicity assume that the radius of curvature of $A d S_{2}$ and the sizes of the other large dimensions labelled by $y$ are of the same order $a$, but this assumption can be easily relaxed. The Euclidean near horizon $(d+2)$ dimensional metric $g_{\mu \nu}$ can then be written as

$$
g_{\mu \nu} d z^{\mu} d z^{\nu}=a^{2} f(y)\left(d \eta^{2}+\sinh ^{2} \eta d \theta^{2}\right)+a^{2} d s_{K}^{2},
$$

where $(\eta, \theta)$ are the coordinates along $A d S_{2}, f(y)$ is some function of the coordinates $y$ along $K$, and $d s_{K}^{2}$ is constructed out of differentials which are invariant under the $S L(2, R)$ isometry of $A d S_{2}$. Note that we have explicitly factored out the large dimensional parameter $a^{2}$ so that $f(y)$ and the metric $d s_{K}^{2}$ are of order unity. Some examples of such metrics can be found in [62] (in a different coordinate system for labelling $A d S_{2}$ metric). It follows from the $S L(2, R)$ isometry of $A d S_{2}$ that

$$
\sqrt{\operatorname{det} g}=\sinh \eta G(y)
$$

for some function $G(y)$ of order $a^{d+2}$. In particular $G(y)$ has no dependence on the coordinates of $A d S_{2}$.

Let $Z_{A d S_{2}}$ denote the partition function of string theory in the near horizon geometry, evaluated by carrying out functional integral over all the string fields weighted by the exponential of the Euclidean action $\mathcal{S}$, with boundary conditions such that asymptotically the field configuration approaches the near horizon geometry of the black hole. Since in $A d S_{2}$ the asymptotic boundary conditions fix the electric fields, or equivalently the charges carried by the black hole, and let the constant modes of the gauge fields fluctuate, we need to include in the path integral a boundary term $\exp \left(-i \oint \sum_{k} q_{k} A_{\mu}^{(k)} d x^{\mu}\right)$ where $A_{\mu}^{(k)}$ are the gauge fields and $q_{k}$ are the corresponding electric charges carried by the black hole [23]. The list of gauge fields include all the gauge fields of the original theory, as well as any gauge field which might arise from dimensional reduction of the metric along the compact directions of the near horizon geometry. Thus we have

$$
Z_{A d S_{2}}=\int d \Psi \exp \left(\mathcal{S}-i \oint \sum_{k} q_{k} A_{\mu}^{(k)} d x^{\mu}\right),
$$

where $\Psi$ stands for all the string fields. $A d S_{2} / C F T_{1}$ correspondence tells us that the full quantum corrected entropy $S_{\text {macro }}$ is related to $Z_{A d S_{2}}$ via [23]:

$$
e^{S_{\text {macro }}-E_{0} L}=Z_{A d S_{2}}
$$


where $E_{0}$ is the energy of the ground state of the black hole carrying a given set of charges, and $L$ denotes the length of the boundary of $A d S_{2}$ in a regularization scheme that renders the volume of $A d S_{2}$ finite by putting an infrared cut-off $\eta \leq \eta_{0}$.

Let $\Delta \mathcal{L}_{\text {eff }}(y)$ denote the quantum correction to the $(d+2)$ dimensional effective lagrangian density evaluated in the background geometry (2.1). Due to the $S L(2, R)$ invariance, $\Delta \mathcal{L}_{\text {eff }}$ is independent of the $A d S_{2}$ coordinates $x$ but could depend on the coordinates $y$ of the large compact dimensions. Then the quantum correction to $Z_{A d S_{2}}$ is given by

$$
\exp \left[\int \sqrt{\operatorname{det} g} d \eta d \theta d^{d} y \Delta \mathcal{L}_{e f f}\right]=\exp \left[2 \pi\left(\cosh \eta_{0}-1\right) \int d^{d} y G(y) \Delta \mathcal{L}_{e f f}(y)\right] .
$$

The term proportional to $\cosh \eta_{0}$ in the exponent has the interpretation of $-L \Delta E_{0}+\mathcal{O}\left(L^{-1}\right)$ where $L=2 \pi \sinh \eta_{0}$ is the length of the boundary of $A d S_{2}$ parametrized by $\theta$ and $\Delta E_{0}=$ $-\int d^{d} y G(y) \Delta \mathcal{L}_{\text {eff }}(y)$ is the shift in the ground state energy 2 Alternatively, this can be cancelled by a boundary counterterm which gives a contribution proportional to the length of the boundary 1.e. $\sinh \eta_{0}$. The rest of the contribution in the exponent can be interpreted as the quantum correction to the black hole entropy [23]. Thus we have

$$
\Delta S_{\text {macro }}=-2 \pi \int d^{d} y G(y) \Delta \mathcal{L}_{\text {eff }}(y) .
$$

While the term in the exponent proportional to $L$ and hence $\Delta E_{0}$ can get further corrections from boundary terms in the action, the finite part $\Delta S_{\text {macro }}$ is defined unambiguously. This reduces the problem of computing quantum correction to the black hole entropy to that of computing quantum correction to $\mathcal{L}_{\text {eff }}$. If on the other hand we replace $\Delta \mathcal{L}_{\text {eff }}$ in (2.6) by the classical Lagrangian density then we get back the Wald entropy [66] of the classical black hole.

So far our analysis has been completely general without making any approximation. However if we are interested in computing the logarithmic correction to the entropy then we can focus on the contribution to $\Delta \mathcal{L}_{\text {eff }}$ from the massless fields only [25,26]. We shall now describe the general procedure for calculating one loop contribution to $\Delta \mathcal{L}_{\text {eff }}(y)$ from massless fields.

\subsection{Logarithmic correction from the non-zero modes}

Let us suppose that the string theory under consideration contains a set of massless fields $\left\{\phi_{i}\right\}$ (or fields of mass $\sim a^{-1}$ ) on $A d S_{2} \times K$. Here the index $i$ could run over several scalar

\footnotetext{
${ }^{2}$ In order to fix the normalization in the definition of $L$ and $E$ we need to fix the normalization of the metric on $A d S_{2}$ after dimensional reduction on $K$. We can for example take $\left(d \eta^{2}+\sinh ^{2} \eta d \theta^{2}\right)$ as the definition of the metric on $A d S_{2}$ in which case the boundary has length $2 \pi \sinh \eta_{0}$. Any other normalization will simply rescale $E$ and $L$ in opposite directions without affecting the rest of the analysis.
} 
fields, or the space-time indices of tensor fields. Let $f_{n}^{(i)}(x, y)$ denote an orthonormal basis of eigenfunctions of the kinetic operator expanded around the near horizon geometry, with eigenvalue $\kappa_{n}$ :

$$
\int d^{2} x d^{d} y \sqrt{\operatorname{det} g} G_{i j} f_{n}^{(i)}(x, y) f_{m}^{(j)}(x, y)=\delta_{m n},
$$

where $G_{i j}$ is a metric in the space of fields induced by the metric on the near horizon geometry, e.g. for a vector field $A_{\mu}, G^{\mu \nu}=g^{\mu \nu}$. Then the heat kernel $K^{i j}\left(x, y ; x^{\prime}, y^{\prime}\right)$ is defined as

$$
K^{i j}\left(x, y ; x^{\prime}, y^{\prime} ; s\right)=\sum_{n} e^{-\kappa_{n} s} f_{n}^{(i)}(x, y) f_{n}^{(j)}\left(x^{\prime}, y^{\prime}\right)
$$

In (2.7), (2.8) we have assumed that we are working in a basis in which the eigenfunctions are real; if this is not the case then we need to replace one of the $f_{n}^{(i)}$ 's by $f_{n}^{(i) *}$. Among the $f_{n}^{(i)}$ 's there may be a special set of modes for which $\kappa_{n}$ vanishes. We shall denote these zero modes by the special symbol $g_{\ell}^{(i)}(x, y)$, normalized as

$$
\int d^{2} x d^{d} y \sqrt{\operatorname{det} g} G_{i j} g_{\ell}^{(i)}(x, y) g_{\ell^{\prime}}^{(j)}(x, y)=\delta_{\ell \ell^{\prime}}
$$

and define

$$
\begin{gathered}
\bar{K}^{i j}\left(x, y ; x^{\prime}, y^{\prime}\right)=\sum_{\ell} g_{\ell}^{(i)}(x, y) g_{\ell}^{(j)}\left(x^{\prime}, y^{\prime}\right), \\
K(y ; s)=G_{i j} K^{i j}(x, y ; x, y ; s), \quad \bar{K}(y)=G_{i j} \bar{K}^{i j}(x, y ; x, y) .
\end{gathered}
$$

Note that due to the $S L(2, R)$ symmetry, $G_{i j} K^{i j}(x, y ; x, y ; s)$ and $G_{i j} \bar{K}^{i j}(x, y ; x, y)$ depend only on $y$ but not on $x$. Using orthonormality of the wave-functions we get

$$
\int d^{2} x d^{d} y \sqrt{\operatorname{det} g}(K(y ; s)-\bar{K}(y))=\sum_{n}^{\prime} e^{-\kappa_{n} s}
$$

where $\sum_{n}^{\prime}$ denotes sum over the non-zero modes only. Since the one loop contribution to $Z_{A d S_{2}}$ from the non-zero modes is given by $\prod_{n}^{\prime} \kappa_{n}^{-1 / 2}=\exp \left[-\frac{1}{2} \ln \kappa_{n}\right]$, the contribution to the one loop effective action can now be expressed as

$$
\Delta \mathcal{S}=-\frac{1}{2} \sum_{n}^{\prime} \ln \kappa_{n}=\frac{1}{2} \int_{\epsilon}^{\infty} \frac{d s}{s} \sum_{n}^{\prime} e^{-\kappa_{n} s}=\frac{1}{2} \int_{\epsilon}^{\infty} \frac{d s}{s} \int d^{2} x d^{d} y \sqrt{\operatorname{det} g}(K(y ; s)-\bar{K}(y)),
$$

where $\epsilon$ is an ultraviolet cut-off which we shall take to be of order unity, 1.e. string scale. Identifying (2.13) as the contribution to $\int d^{2} x d^{d} y \sqrt{\operatorname{det} g} \Delta \mathcal{L}_{\text {eff }}(y)$ we get the contribution to $\Delta \mathcal{L}_{\text {eff }}(y)$ from the non-zero modes:

$$
\Delta \mathcal{L}_{e f f}^{(n z)}(y)=\frac{1}{2} \int_{\epsilon}^{\infty} \frac{d s}{s}(K(y ; s)-\bar{K}(y)) .
$$


Substituting this into (2.6) we get the one loop contribution to $\Delta S_{\text {macro }}$ due to the non-zero modes:

$$
\Delta S_{\text {macro }}=-\pi \int_{\epsilon}^{\infty} \frac{d s}{s} \int d^{d} y G(y)(K(y ; s)-\bar{K}(y)) .
$$

Since we have labelled by $a^{2}$ the overall scale factor in the metric, the non-zero eigenvalues $\kappa_{n}$ of the kinetic operator scale as $1 / a^{2}$ and hence $K(y, s)-\bar{K}(y)$ is a function of $\bar{s} \equiv s / a^{2}$. Thus it is more natural to express (2.15) as

$$
\Delta S_{\text {macro }}=-\pi \int_{\epsilon / a^{2}}^{\infty} \frac{d \bar{s}}{\bar{s}} \int d^{d} y G(y)(K(y ; s)-\bar{K}(y)) .
$$

In this case the logarithmic contribution to the entropy - term proportional to $\ln a$ - arises from the $\epsilon / a^{2}<<\bar{s}<<1$, 1.e. $\epsilon<<s<<a^{2}$ region in the $s$ integral 3 If we expand $K(y ; s)$ in a Laurent series expansion in $\bar{s}=s / a^{2}$ in the region $\bar{s}<<1$, and if $K_{0}(y)$ denotes the coefficient of the constant mode in this expansion, then using (2.16) we see that the net logarithmic correction to the entropy from the non-zero modes will be given by

$$
-2 \pi \ln a \int d^{d} y G(y)\left(K_{0}(y)-\bar{K}(y)\right) .
$$

\subsection{Zero mode contribution}

The contribution to $Z_{A d S_{2}}$ from integration over the zero modes can be evaluated as follows. First note that we can use (2.9) $,(2.10),(2.11)$ to define the number $N_{z m}$ of zero modes:

$$
\int d^{2} x d^{d} y \sqrt{\operatorname{det} g} \bar{K}(y)=\sum_{\ell} 1=N_{z m} .
$$

In fact often the matrix $\bar{K}^{i j}$ takes a block diagonal form in the field space, with different blocks representing zero modes of different sets of fields. In that case we can use the analog of (2.18) to define the number of zero modes of each block. If these different blocks are labelled by $\left\{A_{r}\right\}$ and we define

$$
\bar{K}^{r}(y) \equiv \sum_{\ell \in A_{r}} G_{i j} g_{\ell}^{(i)}(x, y) g_{\ell}^{(j)}(x, y),
$$

then the number of zero modes belonging to the $r$-th block will be given by

$$
N_{z m}^{(r)}=\int d^{2} x d^{d} y \sqrt{\operatorname{det} g} \bar{K}^{r}(y)=2 \pi\left(\cosh \eta_{0}-1\right) \int d^{d} y G(y) \bar{K}^{r}(y) .
$$

\footnotetext{
${ }^{3}$ If on the other hand the theory has a cosmological constant, e.g. for BTZ black holes, then the scale of the eigenvalues of the kinetic operator are set by the cosmological constant instead of the parameter $a$. In this case the entire logarithmic corrections would come only from integration over the zero modes.
} 
Typically these zero modes are associated with certain asymptotic symmetries, - gauge transformation with parameters which do not vanish at infinity. In this case we can evaluate the integration over the zero modes by making a change of variables from the coefficients of the zero modes to the parameters labelling the (super-)group of asymptotic symmetries. We shall use an $a$-independent parametrization of the asymptotic symmetry group so that the group composition laws and the range over which the parameters take value is $a$-independent. Suppose for the zero modes in the $r^{\prime}$ th block the Jacobian for the change of variables from the fields to supergroup parameters gives a factor of $a^{\beta_{r}}$ for each zero mode. Then the net $a$ dependent contribution to $Z_{A d S_{2}}$ from the zero mode integration will be given by

$$
a^{\sum_{r} \beta_{r} N_{z m}^{(r)}}=\exp \left[2 \pi \ln a\left(\cosh \eta_{0}-1\right) \int d^{d} y G(y) \sum_{r} \beta_{r} \bar{K}^{r}(y)\right] .
$$

As before we can interpret the term in the exponent proportional to $\cosh \eta_{0}$ as a contribution to the ground state energy and the finite term as a contribution to $\Delta S_{\text {macro }}$. Adding this to (2.17) we get

$$
\Delta S_{\text {macro }}=-2 \pi \ln a \int d^{d} y G(y)\left(K_{0}(y)+\sum_{r}\left(\beta_{r}-1\right) \bar{K}^{r}(y)\right) .
$$

We shall refer to the term proportional to $\sum_{r}\left(\beta_{r}-1\right) \int d^{d} y G(y) \bar{K}^{r}(y)$ as the zero mode contribution although it should be kept in mind that only the term proportional to $\beta_{r}$ arises from integration over the zero modes, and the -1 term is the result of subtracting the zero mode contribution from the heat kernel to correctly compute the result of integration over the non-zero modes.

The contribution from the fermionic fields can be included in the above analysis as follows. Let $\left\{\psi^{i}\right\}$ denote the set of fermion fields in the theory. Here $i$ labels the internal indices or space-time vector index (for the gravitino fields) but the spinor indices are suppressed. Without any loss of generality we can take the $\psi^{i}$ 's to be Majorana spinors satisfying $\bar{\psi}^{i}=\left(\psi^{i}\right)^{T} \widetilde{C}$ where $\widetilde{C}$ is the charge conjugation operator. Then the kinetic term for the fermions have the form

$$
-\frac{1}{2} \bar{\psi}^{i} \mathcal{D}_{i j} \psi^{j}=-\frac{1}{2}\left(\psi^{i}\right)^{T} \widetilde{C} \mathcal{D}_{i j} \psi^{j},
$$

for some appropriate operator $\mathcal{D}$. We can now proceed to define the heat kernel of the fermions in terms of eigenvalues of $\mathcal{D}$ in the usual manner, but with the following simple changes. Since the integration over the fermions produce $(\operatorname{det} \mathcal{D})^{1 / 2}$ instead of $(\operatorname{det} \mathcal{D})^{-1 / 2}$, we need to include 
an extra minus sign in the definition of the heat kernel. Also since the fermionic kinetic operator is linear in derivative, it will be convenient to first compute the determinant of $\mathcal{D}^{2}$ and then take an additional square root of the determinant. This is implemented by including an extra factor of $1 / 2$ in the definition of the heat kernel. We shall denote by $K_{0}^{f}(y)$ the $s$-independent part of the trace of the fermionic heat kernel in the small $s$ expansion after taking into account this factor of $-1 / 2$. To identify the zero modes however we need to work with the kinetic operator and not its square since the zero mode structure may get modified upon taking the square e.g. the kinetic operator may have blocks in the Jordan canonical form which squares to zero, but the matrix itself may be non-zero 4 Let us denote by $\bar{K}^{f}(y)$ the total fermion zero mode contribution to $K_{0}^{f}(y)$. Then we arrive at an expression similar to (2.17) for the fermionic non-zero mode contribution to the entropy:

$$
-2 \pi \ln a \int d^{d} y G(y)\left(K_{0}^{f}(y)-\bar{K}^{f}(y)\right) .
$$

Next we need to carry out the integration over the zero modes. Taking into account the extra factor of $-1 / 2$ in the definition of the fermionic heat kernel we see that the analog of (2.20) for the total number of fermion zero modes $N_{z m}^{(f)}$ now takes the form

$$
N_{z m}^{(f)}=-4 \pi\left(\cosh \eta_{0}-1\right) \int d^{d} y G(y) \bar{K}^{f}(y) .
$$

Let us further assume that integration over each fermion zero modes gives a factor of $a^{-\beta_{f} / 2}$ for some constant $\beta_{f}$. Then the total $a$-dependent contribution from integration over the fermion zero modes is given by

$$
a^{-\beta_{f} N_{z m}^{(f)} / 2}=\exp \left[2 \pi \ln a\left(\cosh \eta_{0}-1\right) \int d^{d} y G(y) \beta_{f} \bar{K}^{f}(y)\right] .
$$

As usual the coefficient of $\cosh \eta_{0}$ can be interpreted as due to a shift in the energy $E_{0}$, whereas the $\eta_{0}$ independent term has the interpretation of a contribution to the black hole entropy. Combining this with the contribution (2.24) from the non-zero modes we arrive at the following expression for the logarithmic correction to the entropy from the fermion zero modes:

$$
\Delta S_{\text {macro }}=-2 \pi \ln a \int d^{d} y G(y)\left(K_{0}^{f}(y)+\left(\beta_{f}-1\right) \bar{K}^{f}(y)\right) .
$$

\footnotetext{
${ }^{4}$ This problem would not arise if we work with $\widetilde{C} \mathcal{D}$ instead of $\mathcal{D}$ since $\widetilde{C} \mathcal{D}$ is represented by an anti-symmetric matrix. However it is easier to work with $\mathcal{D}^{2}$ instead of $(\widetilde{C} \mathcal{D})^{2}$.
} 
In other words, we can use (2.22) to represent contributions from both the bosonic and the fermionic modes provided we include the extra factors of $-1 / 2$ in the definition of the heat kernel and $\beta_{r}$ for the fermions.

At the end of this process we are still left with an $a$-independent contribution from integration over the supergroup which contains both bosonic and fermonic zero modes. Using supersymmetric localization we can get finite result for this integral by canceling the infinities from the bosonic zero mode integration against the zeroes from the fermion zero mode integration [67,68]. However since there is no $a$-dependence in this contribution we shall not discuss this any further.

\subsection{Evaluation of $K_{0}(y)$}

In [25 27] $K_{0}(y)$ was evaluated for various fields by finding the eigenfunctions and eigenvalues of the kinetic operator in the $A d S_{2} \times S^{2}$ near horizon geometry. Since this is a difficult task in absence of rotational symmetry, we shall now describe an indirect method [69 77$]$ for computing $K_{0}(y)$ which works under special circumstances. It can be argued on general grounds that the small $s$ expansion of $K(y ; s)$ contains even (odd) powers of $s^{1 / 2}$ in even (odd) dimensions (see e.g. [76] for a recent review). As a result $K_{0}(y)$, which is the coefficient of the $s^{0}$ term in the small $s$ expansion of $K(y ; s)$, vanishes in odd dimensions. Thus in the rest of this subsection we shall restrict our analysis to the four dimensional (1.e. $d=2$ ) case. In this case one can show that [69 77] if we have purely gravitational background, e.g. as in the case of extremal Kerr black holes, then in a theory with $n_{S}$ minimally coupled massless scalar fields, $n_{V}$ minimally coupled massless vector fields, $n_{F}$ minimally coupled massless Dirac fields, $n_{3 / 2}$ minimally coupled massless spin $3 / 2$ field and $n_{2}$ minimally coupled massless spin 2 fields, $K_{0}(y)$ is given by

$$
K_{0}(y)=-\frac{1}{90 \pi^{2}}\left(n_{S}+62 n_{V}+11 n_{F}\right) E-\frac{1}{30 \pi^{2}}\left(n_{S}+12 n_{V}+6 n_{F}-\frac{233}{6} n_{3 / 2}+\frac{424}{3} n_{2}\right) I,
$$

where

$$
\begin{aligned}
E & =\frac{1}{64}\left(R_{\mu \nu \rho \sigma} R^{\mu \nu \rho \sigma}-4 R_{\mu \nu} R^{\mu \nu}+R^{2}\right) \\
I & =-\frac{1}{64}\left(R_{\mu \nu \rho \sigma} R^{\mu \nu \rho \sigma}-2 R_{\mu \nu} R^{\mu \nu}+\frac{1}{3} R^{2}\right) .
\end{aligned}
$$

In pure gravity $R_{\mu \nu}$ and $R$ vanish for classical solutions, and (2.28) can be written as

$$
K_{0}(y)=-\frac{1}{90 \pi^{2}}\left(-2 n_{S}+26 n_{V}-7 n_{F}+\frac{233}{2} n_{3 / 2}-424 n_{2}\right) E .
$$


In the presence of background electromagnetic field strength eq.(2.30) gets additional contribution involving powers of the background field strength. In principle one could write down the most general four derivative terms on the right hand side of (2.28) and calculate the coefficients of these terms by perturbative computation of the trace anomaly. Alternatively in supersymmetric theories one could invoke supersymmetry to constrain the terms on the right hand side of (2.28). In particular for theories with $\mathcal{N} \geq 2$ supersymmetry in four dimensions one can argue that (2.30) gives the exact result for $K_{0}(y)$ [27, 73]. In our analysis we shall mainly focus on solutions without any background flux and hence use (2.30).

\subsection{Computation of $\beta_{r}$}

We shall now describe the procedure for computing $\beta_{r}$ for various fields following [27]. Let us begin with the contribution from an $U(1)$ gauge field $A_{\mu}$. The path integral measure over $A_{\mu}$ is normalized via

$$
\int\left[D A_{\mu}\right] \exp \left[-\int d^{d+2} z \sqrt{\operatorname{det} g} g^{\mu \nu} A_{\mu} A_{\nu}\right]=1,
$$

where, as mentioned earlier, $z^{\mu}$ stand for both the coordinates $x$ along $A d S_{2}$ and the coordinates $y$ along the large compact dimensions. In the scaling limit we consider $g_{\mu \nu}$ can be written as $a^{2} g_{\mu \nu}^{(0)}$ where $a$ scales as some power of $\Lambda$ and $g_{\mu \nu}^{(0)}$ is an $a$ independent constant metric. Thus we can express (2.31) as

$$
\int\left[D A_{\mu}\right] \exp \left[-a^{d} \int d^{d+2} z \sqrt{\operatorname{det} g^{(0)}} g^{(0) \mu \nu} A_{\mu} A_{\nu}\right]=1 .
$$

From this we see that up to an $a$ independent normalization constant, $\left[D A_{\mu}\right]$ actually corresponds to integration with measure $\prod_{\mu, z} d\left(a^{d / 2} A_{\mu}(z)\right)$. On the other hand the gauge field zero modes are associated with deformations produced by the gauge transformations with non-normalizable parameters: $\delta A_{\mu} \propto \partial_{\mu} \Lambda(z)$ for some functions $\Lambda(z)$ with $a$-independent integration range. Thus the result of integration over the gauge field zero modes can be found by first changing the integration over the zero modes of $\left(a^{d / 2} A_{\mu}\right)$ to integration over $\Lambda$ and then picking up the contribution from the Jacobian in this change of variables. This gives a factor of $a^{d / 2}$ from integration over each zero mode of $A_{\mu}$. Comparing this with the definition of $\beta_{r}$ given above (2.21) we see that for gauge fields we have $\beta_{v}=d / 2$.

The effect of integration over the zero modes of the fluctuations $h_{\mu \nu}$ of the metric (including those of the gauge fields arising from the dimensional reduction of the metric along the large 
compact dimensions) can be found in a similar way, with (2.31), (2.32) replaced by

$$
\int\left[D h_{\mu \nu}\right] \exp \left[-\int d^{d+2} z \sqrt{\operatorname{det} g} g^{\mu \nu} g^{\rho \sigma} h_{\mu \rho} h_{\nu \sigma}\right]=1,
$$

1.e.

$$
\int\left[D h_{\mu \nu}\right] \exp \left[-a^{d-2} \int d^{d+2} z \sqrt{\operatorname{det} g^{(0)}} g^{(0) \mu \nu} g^{(0) \rho \sigma} h_{\mu \rho} h_{\nu \sigma}\right]=1 .
$$

Thus the correctly normalized integration measure, up to an $a$ independent constant, is $\prod_{z,(\mu \nu)} d\left(a^{(d-2) / 2} h_{\mu \nu}(z)\right)$. Now the zero modes of the metric are associated with diffeomorphisms with non-normalizable parameters: $h_{\mu \nu} \propto D_{\mu} \xi_{\nu}+D_{\nu} \xi_{\mu}$, with the diffeomorphism parameter $\xi^{\mu}(z)$ having $a$ independent integration range. Thus the $a$ dependence of the integral over the metric zero modes can be found by finding the Jacobian from the change of variables from $a^{(d-2) / 2} h_{\mu \nu}$ to $\xi^{\mu}$. Lowering of the index of $\xi^{\mu}$ gives a factor of $a^{2}$, leading to a factor of $a^{(d+2) / 2}$ per zero mode. Thus for the metric we have $\beta_{m}=(d+2) / 2$.

Next we turn to the contribution due to the zero modes of the gravitino field $\psi_{\mu}$. In this case eqs.(2.31), (2.32) are replaced by:

$$
\int\left[D \psi_{\mu}\right] \exp \left[-\int d^{d+2} z \sqrt{\operatorname{det} g} g^{\mu \nu} \bar{\psi}_{\mu} \bar{\psi}_{\nu}\right]=1
$$

1.e.

$$
\int\left[D \psi_{\mu}\right] \exp \left[-a^{d} \int d^{d+2} z \sqrt{\operatorname{det} g^{(0)}} g^{(0) \mu \nu} \bar{\psi}_{\mu} \bar{\psi}_{\nu}\right]=1 \text {. }
$$

Thus up to an $a$ independent normalization constant $\left[D \psi_{\mu}\right]$ stands for $\prod d\left(a^{d / 2} \psi_{\mu}\right)$. On the other hand these zero modes are associated with the deformations corresponding to local supersymmetry transformation $\left(\delta \psi_{\mu} \propto D_{\mu} \epsilon\right)$ with supersymmetry transformation parameters $\epsilon$ which do not vanish at infinity. Now since the anti-commutator of two supersymmetry transformations correspond to a general coordinate transformation with parameter $\xi^{\mu}=\bar{\epsilon} \gamma^{\mu} \epsilon$, and since $\gamma^{\mu} \sim a^{-1}$, we conclude that $\epsilon_{0}=a^{-1 / 2} \epsilon$ provides a parametrization of the asymptotic supergroup in which the group composition laws become $a$-independent. Writing $\delta\left(a^{d / 2} \psi_{\mu}\right) \propto$ $a^{(d+1) / 2} D_{\mu} \epsilon_{0}$, using the fact that the integration over the supergroup parameter $\epsilon_{0}$ produces an $a$ independent result and that $d\left(\lambda \epsilon_{0}\right)=\lambda^{-1} d \epsilon_{0}$ for a grassmann variable $\epsilon_{0}$, we now see that each fermion zero mode integration produces a factor of $a^{-(d+1) / 2}$. Comparing this with the definition of $\beta_{f}$ given below (2.25) we see that $\beta_{f}=d+1$.

The results of this subsection can be summarized in the relations

$$
\beta_{v}=\frac{d}{2}, \quad \beta_{m}=\frac{d+2}{2}, \quad \beta_{f}=d+1 .
$$

For $d=2$ this reproduces the results of [27]. 


\subsection{Computation of $\int d^{d} y G(y) \bar{K}^{r}(y)$}

Finally we shall discuss the computation of $\int d^{d} y G(y) \bar{K}^{r}(y)$ for various fields. This is best done with the help of (2.20), (2.25) and the results in appendix A. For each massless gauge field on $A d S_{2}$ the number of zero modes is given by eq. (A.5) and for each massless symmetric tensor field on $A d S_{2}$ the number of zero modes is given by (A.8). Comparing these with (2.20) we see that each gauge field on $A d S_{2}$ contributes a factor of 1 to $2 \pi \int d^{d} y G(y) \bar{K}^{r}(y)$ and each symmetric rank two tensor field in $A d S_{2}$ contributes a factor of 3 to $2 \pi \int d^{d} y G(y) \bar{K}^{r}(y)$. Thus for example if we consider the extremal Kerr black hole in four dimensions whose near horizon geometry has a squashed sphere with $\mathrm{U}(1)$ symmetry besides the $A d S_{2}$ factor, we get a $\mathrm{U}(1)$ gauge field and a symmetric rank two tensor field on $A d S_{2}$ after we dimensionally reduce the metric on the squashed sphere. This gives a net contribution of $1+3=4$ to $2 \pi \int d^{d} y G(y) \bar{K}^{r}(y)$. Finally for computing $\int d^{d} y G(y) \bar{K}^{f}(y)$ for BMPV black holes we can use the result (A.20) for the total number of fermion zero modes. Identifying this with (2.25) we get $-4 \pi \int d^{d} y G(y) \bar{K}^{f}(y)=8$. We can test this for quarter BPS black holes in four dimensional $\mathcal{N}=4$ supersymmetric string theories or half BPS black holes in four dimensional $\mathcal{N}=2$ supersymmetric string theories which have identical number of fermion zero modes in the near horizon geometry. For these cases we have $d=2, \beta_{f}=3$ and the net contribution of the fermion zero modes to $\Delta S_{\text {macro }}$ computed from (2.27) will be $8 \ln a$. This agrees with the results of [26,27].

\section{Extremal Kerr black hole in four dimensions}

We now turn to the analysis of logarithmic corrections to the entropy of an extremal Kerr black hole solution in Einstein gravity. We take the gravitational part of the action to be

$$
\mathcal{S}=\int d^{4} x \sqrt{-\operatorname{det} g} \mathcal{L}, \quad \mathcal{L}=R .
$$

We shall assume that the theory has, besides the metric, $n_{S}$ minimally coupled massless scalars, $n_{V}$ minimally coupled massless vector fields, $n_{F}$ minimally coupled massless Dirac fermions and $n_{3 / 2}$ minimally coupled massless Majorana Rarita-Schwinger fields. The near horizon geometry of an extremal Kerr black hole in this theory is given by (see e.g. [61,62] where the near horizon 
solution is written down in somewhat different coordinate systems)

$$
d s^{2}=a^{2}\left(1+\cos ^{2} \psi\right)\left\{-\left(r^{2}-1\right) d t^{2}+d r^{2} /\left(r^{2}-1\right)+d \psi^{2}\right\}+4 a^{2} \frac{\sin ^{2} \psi}{1+\cos ^{2} \psi}(d \phi-(r-1) d t)^{2}
$$

where $(\phi, \psi)$ label the azimuthal and the polar coordinates, $(r, t)$ denote the radial and the time coordinates and $a$ is a constant related to the angular momentum $J$ via the relation

$$
J=16 \pi a^{2}
$$

The classical Bekenstein-Hawking entropy of the black hole, obtained as $1 / 4 G_{N}=4 \pi$ times the area of the event horizon, is given by

$$
S_{B H}=8 \pi a^{2} \int_{0}^{2 \pi} d \phi \int_{0}^{\pi} \sin \psi d \psi=32 \pi^{2} a^{2}=2 \pi J
$$

In order to compute the logarithmic correction to the entropy we first write down the Euclidean near horizon geometry by replacing $t$ by $-i \theta$. We also introduce the new radial coordinate $\eta=\cosh ^{-1} r$ for convenience. This gives

$$
d s^{2}=a^{2}\left(1+\cos ^{2} \psi\right)\left(d \eta^{2}+\sinh ^{2} \eta d \theta^{2}+d \psi^{2}\right)+4 a^{2} \frac{\sin ^{2} \psi}{1+\cos ^{2} \psi}(d \phi+i(\cosh \eta-1) d \theta)^{2} .
$$

Substituting this into eq.(2.2) we get

$$
G(y)=2 a^{4} \sin \psi\left(1+\cos ^{2} \psi\right) .
$$

Using (2.22), (2.27) and carrying out the $\phi$ integral we now get

$$
\Delta S_{\text {macro }}=-8 \pi^{2} a^{4} \ln a \int d \psi \sin \psi\left(1+\cos ^{2} \psi\right)\left(K_{0}(\psi)+\sum_{r}\left(\beta_{r}-1\right) \bar{K}^{r}(\psi)\right),
$$

where the sum over $r$ runs over the bosonic as well as the fermionic fields.

We can now use (2.30) for computing $K_{0}(\psi)$. In this case $R_{\mu \nu}=0, R=0$ and [78, 79]

$$
R_{\mu \nu \rho \sigma} R^{\mu \nu \rho \sigma}=\frac{48}{a^{4}} \frac{1}{\left(1+\cos ^{2} \psi\right)^{6}}\left(1-15 \cos ^{2} \psi+15 \cos ^{4} \psi-\cos ^{6} \psi\right)
$$

This gives

$$
\begin{aligned}
K_{0}(\psi)= & \frac{1}{120 \pi^{2} a^{4}}\left(2 n_{S}-26 n_{V}+7 n_{F}-\frac{233}{2} n_{3 / 2}+424\right) \\
& \times \frac{1}{\left(1+\cos ^{2} \psi\right)^{6}}\left(1-15 \cos ^{2} \psi+15 \cos ^{4} \psi-\cos ^{6} \psi\right) .
\end{aligned}
$$


Substituting this into (3.7) and using the result

$$
\int_{0}^{\pi} d \psi \sin \psi\left(1+\cos ^{2} \psi\right)^{-5}\left(1-15 \cos ^{2} \psi+15 \cos ^{4} \psi-\cos ^{6} \psi\right)=-\frac{1}{6},
$$

we get the non-zero mode contribution to the black hole entropy to be:

$$
-8 \pi^{2} a^{4} \ln a \int d \psi \sin \psi\left(1+\cos ^{2} \psi\right) K_{0}(\psi)=\frac{1}{90} \ln a\left(2 n_{S}-26 n_{V}+7 n_{F}-\frac{233}{2} n_{3 / 2}+424\right) .
$$

This coincides with the results in [47, 58, 59].

The contribution from the zero modes can be computed as follows. Possible zero modes in this case arise from the gauge fields and the metric, - since the black hole is non-supersymmetric there are no gravitino zero modes. Since here $d=2$, (2.37) gives $\beta_{v}=1$ and $\beta_{m}=2$ [27]. Eq.(2.22) now shows that the contribution from the gauge field zero modes, being proportional to $\left(\beta_{v}-1\right)$, vanishes. To find the contribution from the metric zero modes we note that since the near horizon geometry has $\mathrm{U}(1)$ rotational symmetry, the dimensional reduction of the metric along the $(\psi, \phi)$ direction gives a $U(1)$ gauge field and a massless symmetric rank 2 tensor field on $A d S_{2}$. According to the discussion in $\$ 2.6$ we get a net contribution of $(1+3)=4$ to $2 \pi \int d^{d} y G(y) \bar{K}^{m}(y)$. The second term in eq.(2.22) now gives a net contribution of $-4 \ln a$ to $S_{\text {macro }}$. Adding this to the non-zero mode contribution (3.11) we get the net contribution to $\Delta S_{\text {macro }}$ to be

$$
\begin{aligned}
& \frac{1}{90} \ln a\left(2 n_{S}-26 n_{V}+7 n_{F}-\frac{233}{2} n_{3 / 2}+64\right) \\
= & \frac{1}{180} \ln A_{H}\left(2 n_{S}-26 n_{V}+7 n_{F}-\frac{233}{2} n_{3 / 2}+64\right),
\end{aligned}
$$

where $A_{H} \propto a^{2}$ is the area of the event horizon.

Let us denote by $C_{t o t}$ the net coefficient of the $\ln A_{H}$ term appearing in (3.12). In order to seek a microscopic explanation of this result we need to specify for which ensemble (3.12) gives the logarithmic correction to the entropy. As discussed in the introduction, this gives logarithmic correction to $\ln \widetilde{d}(J), \widetilde{d}(J)$ being number that counts all states with fixed $J_{3}=J$ and all gauge charges set to 0 , but no restriction on $\vec{J}^{2}$. We can extract from this the number $d(J)$ where $\vec{J}^{2}$ is also fixed to be $J(J+1)$ as follows. First note that while $\widetilde{d}(J)$ counts all states with fixed $J_{3}=J$ and $\vec{J}^{2} \geq J(J+1), \widetilde{d}(J+1)$ counts all states with fixed $J_{3}$ and $\overrightarrow{J^{2}} \geq(J+1)(J+2)$. Furthermore due to $S U(2)$ symmetry, once we fix $\overrightarrow{J^{2}}$, the index is independent of the chosen value of $J_{3}$, and hence in both cases we can take $J_{3}=J$. Thus 
$\widetilde{d}(J)-\widetilde{d}(J+1)$ will count all states with $\vec{J}^{2}=J(J+1)$ and $J_{3}=J$. This is the desired microscopic index $d(J)$. Thus we have

$$
d(J)=\widetilde{d}(J+1)-\widetilde{d}(J)=e^{S_{B H}(J+1)+C_{t o t} \ln A_{H}+\cdots}-e^{S_{B H}(J)+C_{t o t} \ln A_{H}+\cdots},
$$

where $S_{B H}(J)=2 \pi J$ is the classical Bekenstein-Hawking entropy given in (3.4) and $\cdots$ denote terms of order unity. It is easy to see using (3.4) that the right hand side is given by $\left(e^{2 \pi}-1\right)$ times $\widetilde{d}(J)$. Thus the logarithmic correction to $\ln d(J)$ is the same as that for $\ln \widetilde{d}(J)$, and is given by (3.12).

It will be interesting to explore if Kerr/CFT correspondence [22] or any other approach could give us a microscopic explanation of (3.12).

\section{BMPV black holes}

In this section we shall analyze logarithmic corrections to the entropy of a five dimensional BMPV black hole in type IIB string theory on $K 3 \times S^{1}$, as well as in a class of CHL models [33 36] obtained by taking $\mathbb{Z}_{\mathbf{N}}$ orbifolds of type IIB string theory on $K 3 \times S^{1}$. The $\mathbb{Z}_{\mathbf{N}}$ transformation acts by $2 \pi / \mathbf{N}$ shift along $S^{1}$ and an appropriate action on $K 3$ that preserves 16 supersymmetries. Our analysis also holds for BMPV black holes in type IIB string theory on $T^{5}$ and its various orbifolds discussed in [19,20] with the only change that the metric $\widehat{g}_{m n}$ in (4.2) represents metric on $T^{4}$. This macroscopic result for logarithmic correction to the entropy will then be compared with the microscopic results derived in $\$ 5$,

Some recent discussion on higher derivative corrections to five dimensional black hole entropy can be found in [31,80,89]. The results of these papers imply agreement between macroscopic and microscopic entropy at the leading order $\left(\right.$ charge $\left.^{3 / 2}\right)$ and the first subleading order $\left(\right.$ charge $\left.^{1 / 2}\right)$. The logarithmic corrections to be studied here constitute the next leading correction to the entropy.

\subsection{The near horizon geometry}

In the $\alpha^{\prime}=1$ unit the ten dimensional action of type IIB string theory takes the form

$$
\begin{aligned}
S & =\int d^{10} x \sqrt{-\operatorname{det} G} \mathcal{L} \\
\mathcal{L} & =\frac{1}{(2 \pi)^{7}}\left[e^{-2 \Phi}\left(R+4 G^{M N} \partial_{M} \Phi \partial_{N} \Phi\right)-\frac{1}{12} F_{M N P} F^{M N P}\right]
\end{aligned}
$$


where $G_{M N}$ is the ten dimensional string metric, $\Phi$ is the dilaton and $F_{M N P}$ is the RR 3-form field strength under which the D1 and D5 branes are electrically and magnetically charged. We shall choose the coordinate along $S^{1}$ such that $S^{1} / \mathbb{Z}_{\mathbf{N}}$ has period $2 \pi R_{5}$, and as we move $2 \pi R_{5}$ along this direction we come back to the same point on the circle but a $\mathbb{Z}_{\mathbf{N}}$ transformed point on $K 3$. A unit momentum will be defined as $1 / R_{5}$. Since there are four non-compact space directions, this theory has an $S O(4)=S U(2)_{L} \times S U(2)_{R}$ rotational symmetry. We shall denote by $J_{i L}$ and $J_{i R}$ for $i=1,2,3$ the generators of the $S U(2)_{L}$ and $S U(2)_{R}$ rotation groups. In this theory we consider a classical BPS black hole solution carrying $Q_{5}$ units of D5-brane charge along $K 3 \times S^{1}, Q_{1}$ units of D1-brane charge along $S^{1}$ (including the $-Q_{5}$ units of D1-brane charge that is induced by wrapping $Q_{5}$ D5-branes on $\left.K 3\right),-n / \mathbf{N}=-\tilde{n}$ units of momentum along $S^{1} / \mathbb{Z}_{\mathbf{N}}, J / 2$ units of $J_{3 L}$ charge and zero $J_{1 L}, J_{2 L}$ and $J_{i R}$ charge [2]. The Lorentzian ten dimensional near horizon geometry takes the form [90, 91]

$$
\begin{aligned}
d S^{2}= & r_{0} \frac{d \rho^{2}}{\rho^{2}}+d \chi^{2}+r_{0}\left(d x^{4}+\cos \psi d \phi\right)^{2}+\frac{\widetilde{J}}{4 r_{0}} d \chi\left(d x^{4}+\cos \psi d \phi\right)-2 \rho d \chi d \tau \\
& +r_{0}\left(d \psi^{2}+\sin ^{2} \psi d \phi^{2}\right)+\widehat{g}_{m n} d u^{m} d u^{n}, \\
e^{\Phi}= & \lambda \\
F= & \frac{r_{0}}{\lambda}\left[\epsilon_{3}+* \epsilon_{3}+\frac{\widetilde{J}}{8 r_{0}^{2}} d \chi \wedge\left(\frac{1}{\rho} d \rho \wedge\left(d x^{4}+\cos \psi d \phi\right)+\sin \psi d \psi \wedge d \phi\right)\right], \\
& \left(\psi, \phi, x^{4}\right) \equiv\left(2 \pi-\psi, \phi+\pi, x^{4}+\pi\right) \equiv\left(\psi, \phi+2 \pi, x^{4}+2 \pi\right) \equiv\left(\psi, \phi, x^{4}+4 \pi\right), \\
& (\chi, \vec{u}) \equiv\left(\chi+2 \pi R_{5}, h \vec{u}\right) .
\end{aligned}
$$

Here $u_{m}$ are coordinates along $K 3, \widehat{g}_{m n}$ is the metric along $K 3, h$ represents the action of the $\mathbb{Z}_{\mathbf{N}}$ generator on the coordinates of $K 3, \lambda$ is an arbitrary constant, $\epsilon_{3}=\sin \psi d x^{4} \wedge d \psi \wedge d \phi$ denotes the volume form on a 3-sphere of coordinate radius 2 labelled by the coordinates $\left(x^{4}, \psi, \phi\right)$ and $*$ denotes Hodge dual in six dimensions spanned by the coordinates $t, \chi, \rho, x^{4}$, $\psi$ and $\phi$. The constants $r_{0}, R_{5}, \widetilde{J}$ and the volume $V$ of $K 3$ are determined in terms of the charges via the relations

$$
r_{0}=\frac{\lambda Q_{5}}{4}, \quad R_{5}=\sqrt{\frac{\lambda \widetilde{n}}{Q_{1}}}, \quad \widetilde{J}=\frac{J}{2} \frac{Q_{5}}{\sqrt{Q_{1} \widetilde{n}}} \lambda^{3 / 2}, \quad V \equiv \int d^{4} u \sqrt{\operatorname{det} \widehat{g}}=(2 \pi)^{4} \frac{Q_{1}}{Q_{5}} .
$$

The Bekenstein-Hawking entropy of this black hole can be computed by dividing the area of the event horizon, spanned by the coordinates $\left(\chi, x^{4}, \theta, \phi, \vec{u}\right)$, by four times the effective Newton's 
constant at the horizon, read out from (4.1)-(4.3). The result is:

$$
S_{B H}=2 \pi \sqrt{Q_{1} Q_{5} \widetilde{n}-\frac{J^{2}}{4}} .
$$

In order to make the $S L(2, R)$ symmetry of $A d S_{2}$ manifest, we define

$$
A=\sqrt{r_{0}}\left(1-\frac{\widetilde{J}^{2}}{64 r_{0}^{3}}\right)^{-1 / 2}, \quad B=-\frac{\widetilde{J}}{8 r_{0}^{2}} A
$$

and change coordinates to

$$
\widetilde{\tau}=A \tau / r_{0}
$$

In these coordinates the near horizon metric takes the form

$$
\begin{aligned}
d S^{2}= & r_{0} \frac{d \rho^{2}}{\rho^{2}}-r_{0} \rho^{2} d \widetilde{\tau}^{2}+(d \chi-A \rho d \widetilde{\tau})^{2}+r_{0}\left(d x^{4}+\cos \psi d \phi-B \rho d \widetilde{\tau}\right)^{2}+r_{0}\left(d \psi^{2}+\sin ^{2} \psi d \phi^{2}\right) \\
& +\frac{\widetilde{J}}{4 r_{0}}(d \chi-A \rho d \widetilde{\tau})\left(d x^{4}+\cos \psi d \phi-B \rho d \widetilde{\tau}\right)+\widehat{g}_{m n} d u^{m} d u^{n}
\end{aligned}
$$

This metric has an $S L(2, R)$ isometry generated by [61]

$$
L_{1}=\partial_{\tilde{\tau}}, \quad L_{0}=\widetilde{\tau} \partial_{\widetilde{\tau}}-\rho \partial_{\rho}, \quad L_{-1}=(1 / 2)\left(1 / \rho^{2}+\widetilde{\tau}^{2}\right) \partial_{\widetilde{\tau}}-(\widetilde{\tau} \rho) \partial_{\rho}+(A / \rho) \partial_{\chi}+(B / \rho) \partial_{x^{4}}
$$

In order to make connection with the coordinate systems used in (2.1) we need to make a coordinate change

$$
\cosh \eta=\frac{1}{2}\left(\rho+\rho^{-1}-\rho \widetilde{\tau}^{2}\right), \quad e^{-2 t}=\frac{(1-\widetilde{\tau})^{2}-\rho^{-2}}{(1+\widetilde{\tau})^{2}-\rho^{-2}}
$$

together with some $(\rho, \widetilde{\tau})$ dependent shifts on the coordinates $\chi$ and $x^{4}$ and then make the analytic continuation $t \rightarrow-i \theta$. The classical entropy function method [66], applied to the near horizon geometry with the action (4.1), give the same result for the entropy as (4.4) as expected [90].

The scaling limit we shall consider is

$$
Q_{1} \sim \Lambda, \quad Q_{5} \sim \Lambda, \quad \widetilde{n} \sim \Lambda, \quad J \sim \Lambda^{3 / 2},
$$

with $\Lambda$ large. Also we shall keep the undetermined constant $\lambda$ fixed as we take the large charge limit. In this limit $r_{0}$ grows as $\Lambda, \widetilde{J}$ grows as $\Lambda^{3 / 2}, R_{5}$ and the volume $V$ of $K 3$ remains fixed, 
and the size $a$ of $A d S_{2}$ spanned by $(\rho, \widetilde{\tau})$ as well as the the size of the squashed 3 -sphere labelled by $\left(x^{4}, \psi, \phi\right)$ grow as

$$
a \equiv \sqrt{r_{0}} \sim \Lambda^{1 / 2}
$$

Thus in this situation we can apply the formalism developed in 92 for computing logarithmic correction to the entropy. For this we dimensionally reduce the theory on the five directions spanned by the coordinates $(\vec{u}, \chi)$ and regard this as a five dimensional theory living on the space spanned by the coordinates $\left(x^{4}, \psi, \phi, \rho, \widetilde{\tau}\right)$. We also dualize the 3 -form field strength $F_{M N P}$ as well as all other 3 -form field strengths to 2-form field strengths so that we can apply the formalism of $\$ 2$.

\subsection{Ensemble choice and index from entropy}

In this subsection we shall make some comments on the ensemble in which we compute the entropy. As argued in [23], the quantum entropy function computes the entropy in the microcanonical ensemble in which all charges and angular momenta, which have the interpretation of charges associated with gauge fields in $A d S_{2}$, are fixed. This means in particular that for the BMPV black hole, $J_{i R}=0$ and $J_{3 L}=J / 2$. This is exactly analogous to the situation for extremal Kerr black hole as discussed at the end of $\$ 3$. On the other hand for the Strominger Vafa black hole carrying zero angular momentum all components of $\vec{J}_{L}$ are associated with gauge charges on $A d S_{2}$ and hence we have $J_{i R}=0$ and $J_{i L}=0$.

Now while comparing our result with the microscopic results we need to use a protected index instead of the degeneracy $[31,32,37,91,92$. In the present situation we can consider two different indices. One of them will be defined as

$$
d\left(n, Q_{1}, Q_{5}, J\right) \equiv-\frac{1}{p !} \operatorname{Tr}\left[(-1)^{2 J_{3 R}}\left(2 J_{3 R}\right)^{p}\right]
$$

where $p$ takes the value 2 for type IIB string theory on $K 3 \times S^{1}$ and the CHL models, but is 6 for type IIB string theory on $T^{5} 5$ The trace is taken over all states carrying fixed $Q_{1}, Q_{5}, n$ and $J_{3 L}=J / 2, \vec{J}_{L}^{2}=\frac{J}{2}\left(\frac{J}{2}+1\right)$ but different values of $J_{3 R}$ and $\vec{J}_{R}^{2}$. The second index will be

\footnotetext{
${ }^{5}$ This agrees with that used in [31,91 but apparently differs from those in the earlier papers e.g. 32, 37. For example for type IIB on $K 3 \times S^{1}$, 32 would have $p=0$ whereas for type IIB on $T^{5}$, 37, would have $p=2$. This difference can be attributed to the fact that the trace in (4.12) is taken over all the modes of the system, whereas in the definition of the index given in [32,37] the trace over the D1-D5 center of mass modes was factored out. The definition we are using is in the same spirit as the helicity trace index used in 93, 94. for four dimensional black holes.
} 
defined as

$$
\widetilde{d}\left(n, Q_{1}, Q_{5}, J\right) \equiv-\frac{1}{p !} \widetilde{\operatorname{Tr}}\left[(-1)^{2 J_{3 R}}\left(2 J_{3 R}\right)^{p}\right]
$$

where $\widetilde{T r}$ denotes that while taking the trace we sum over all states with fixed $Q_{1}, Q_{5}, n$ and $J_{3 L}=J / 2$, but different values of $J_{3 R}, \vec{J}_{R}^{2}$ and $\vec{J}_{L}^{2}$. The difference between the two indices is that the $\vec{J}_{L}^{2}$ value is not fixed in the second index. The two indices are related by a formula similar to (3.13):

$$
d\left(n, Q_{1}, Q_{5}, J\right)=\widetilde{d}\left(n, Q_{1}, Q_{5}, J\right)-\widetilde{d}\left(n, Q_{1}, Q_{5}, J+2\right)
$$

It has been argued in detail in [23, 31] that on the black hole side the exponential of the entropy in fact computes an index. This essentially follows from factoring the trace in (4.12) or (4.13) into a trace over the horizon degrees of freedom and the trace over the hair modes modes living outside the horizon. In particular the hair modes include a set of $2 p$ fermion zero modes associated with broken supersymmetry which are charged under $\vec{J}_{R}$ but not under $\vec{J}_{L}$. $\operatorname{Tr}\left[(-1)^{2 J_{3 R}}\left(2 J_{3 R}\right)^{p}\right]$ appearing in (4.12) receives a non-zero constant contribution from these modes, but each of the $p$ factors of $2 J_{3 R}$ are needed to prevent the contribution from vanishing. After factoring out trace over these zero modes, we are left with $\operatorname{Tr}(-1)^{2 J_{3 R}}$ with the trace taken over the rest of the modes. Since BPS black holes with four unbroken supersymmetries are forced to have $\vec{J}_{R}=0$ [31], all the states represented by the black hole horizon have $(-1)^{2 J_{3 R}}=1$, and hence the contribution to the index from the degrees of freedom associated with the horizon will be given by $\operatorname{Tr}(1)=e^{S_{\text {macro }}}$. If there are no additional hair modes besides the zero modes mentioned above then this allows us to compare $\exp \left[S_{\text {macro }}\right]$ directly with the microscopic index. A similar argument holds for the index (4.13). Whether one also fixes $\vec{J}_{L}^{2}$ or not depends on the situation; for non-zero $J$, the black hole counts states with fixed $J_{3 L}=J / 2$ but all values of $\vec{J}_{L}^{2}$. Thus $\widetilde{d}$ is the relevant index. On the other hand for $J=0$, all components of $\vec{J}_{L}$ are gauge charges and fixed to be zero. Thus the index $d$ is the correct choice.

If there are additional hair modes then we also need to compute their contribution to the index and combine this with the contribution $\exp \left[S_{\text {macro }}\right]$ from the horizon modes. It is however more convenient to identify the macroscopic contribution to the index as the contribution

$$
\exp \left[S_{\text {macro }}\left(n, Q_{1}, Q_{5}, J\right)\right]
$$

from the horizon modes only and remove the contribution of the (macroscopic) hair modes from the microscopic index before comparing the macroscopic and the microscopic results. It 
will be argued in $\$ 5.3$ that in the scaling limit that we are considering here, removal of the hair modes from the microscopic index does not change the coefficient of the logarithmic correction to the entropy and hence (4.15) can be directly compared to the microscopic result for the logarithmic corrections.

\subsection{Logarithmic corrections}

We now describe the macroscopic computation of logarithmic corrections to the BMPV black hole entropy. According to (2.22) this involves three parts: computation of $K_{0}(y)$, computation of $\beta_{r}$ and computation of $\bar{K}^{r}(y)$. As already discussed in $2.4, K_{0}(y)$ vanishes in odd dimensions. Thus we are left with only the contribution from the second term of (2.22).

Let us begin with the contribution from a $U(1)$ gauge field $A_{\mu}$. Eq.(2.37) gives $\beta_{v}=3 / 2$ for $d=3$. On the other hand since each five dimensional gauge field upon dimensional reduction on the squashed $S^{3}$ in the near horizon geometry gives a gauge field on $A d S_{2}$, we have, from \$2.6, that $2 \pi \int d^{d} y G(y) \bar{K}^{v}(y)=1$. Eq.(2.22) now shows that for each gauge field we have a contribution of $-\frac{1}{2} \ln a$ to $\Delta S_{\text {macro }}$. Since $a \sim \Lambda^{1 / 2}$ we see that $n_{V}$ vector fields will give a logarithmic correction of the form $-\left(n_{V} / 4\right) \ln \Lambda$.

The effect of integration over the zero modes of the fluctuations $h_{\mu \nu}$ of the metric (including those of the gauge fields arising from the dimensional reduction of the metric on squashed $S^{3}$ ) can be found in a similar way. Eq.(2.37) gives $\beta_{m}=5 / 2$ for $d=3$. On the other hand since the five dimensional metric leads to $S U(2) \times U(1)$ gauge fields and a metric on $A d S_{2}$ after dimensional reduction on the squashed sphere, we see, after using the results in $\$ 2.6$, that $2 \pi \int d^{d} y G(y) K^{m}(y)=4+3=7$. Eq.(2.22) now shows that the logarithmic correction to $S_{\text {macro }}$ from the five dimensional metric is given by $-(21 / 2) \ln a=-(21 / 4) \ln \Lambda$.

Finally we turn to the contribution due to the zero modes of the gravitino field $\psi_{\mu}$. In this case eq.(2.37) gives $\beta_{f}=4$ for $d=3$. On the other hand the analysis in \$2.6 shows that $-2 \pi \int d^{d} y G(y) \bar{K}^{f}(y)=4$. Eq.(2.27) now shows that the net logarithmic correction to the entropy from the gravitino zero modes is given by $3 \times 4 \ln a=6 \ln \Lambda$.

Combining the contributions from the vector, metric and the gravitino zero modes we get a net logarithmic correction of

$$
\left[-\frac{n_{V}}{4}-\frac{21}{4}+6\right] \ln \Lambda=-\frac{1}{4}\left(n_{V}-3\right) \ln \Lambda .
$$

As already discussed, this logarithmic correction refers to the logarithm of the index $\widetilde{d}\left(n, Q_{1}, Q_{5}, J\right)$ from the macroscopic side. We shall denote this index by $\widetilde{d}_{\text {macro }}\left(n, Q_{1}, Q_{5}, J\right)$. It now follows 
from (4.4) and (4.14) that logarithmic correction to $d_{\text {macro }}\left(n, Q_{1}, Q_{5}, J\right)$ takes the form

$$
d_{\text {macro }}\left(n, Q_{1}, Q_{5}, J\right)=e^{S_{B H}(J)-\frac{1}{4}\left(n_{V}-3\right) \ln \Lambda+\cdots}-e^{S_{B H}(J+2)-\frac{1}{4}\left(n_{V}-3\right) \ln \Lambda+\cdots}=e^{S_{B H}(J)-\frac{1}{4}\left(n_{V}-3\right) \ln \Lambda+\cdots}
$$

when the charges and the angular momentum scale as in (4.10). Here $\cdots$ denote terms of order 1 . In the term on the right hand side we have included an additional factor of order unity, involving the ratio $J / \sqrt{Q_{1} Q_{5} \widetilde{n}} \sim 1$, in the $\cdots$. (4.17) is in perfect agreement with the microscopic result (5.22). It is also in agreement with (5.36) for $\alpha=0$ and $n_{V}=27$, the latter being the number of vector fields in type IIB string theory compactified on $T^{5}$. By comparing (4.16) and (4.17) we also see that in this limit the logarithmic corrections to $d$ and $\widetilde{d}$ are identical.

If we take $J=0$ instead of $J \sim \Lambda^{3 / 2}$ then we get two extra massless gauge fields on $A d S_{2}$ from the reduction of the metric on $S^{3}$, giving the total contribution to $2 \pi \int d^{d} y G(y) \bar{K}^{m}(y)$ from the metric to be $6+3=9$. This changes the metric contribution of $-21 / 4$ in (4.16) to $-27 / 4$, and we get the following result for the logarithmic correction to the entropy:

$$
-\frac{1}{4}\left(n_{V}+3\right) \ln \Lambda
$$

Furthermore this now directly computes the logarithmic correction to the index $d\left(n, Q_{1}, Q_{5}, J\right)$. (4.18) is in perfect agreement with the microscopic result (5.26). This is also in agreement with the microscopic result (5.38) for type IIB string theory on $T^{5}$ if we set $n_{V}=27$.

\section{Microscopic analysis of the BMPV black hole entropy}

In this section we shall derive the microscopic formulæ for the index of a black hole in type IIB string theory compactified on $K 3 \times S^{1} / \mathbb{Z}_{\mathbf{N}}$ for $\mathbf{N}=1,2,3,5,7$ in various limits. Here the $\mathbb{Z}_{\mathbf{N}}$ symmetry acts by $2 \pi / \mathbf{N}$ units of translation along $S^{1}$ and by a geometric transformation on $K 3$ that commutes with 16 supercharges. We also repeat the analysis for type IIB string theory on $T^{5}$.

\subsection{Expression for the index in type IIB on $K 3 \times S^{1} / \mathbf{Z}_{\mathbf{N}}$}

As in $₫ 4$ we consider a system of $Q_{5}$ D5-branes wrapped on $K 3 \times S^{1} / \mathbb{Z}_{N}$, carrying $Q_{1}$ units of D1-brane charge wrapped on $S^{1},-n / \mathbf{N}$ units of momentum along $S^{1}$ and $S U(2)_{L}$ angular 
momentum $J_{3 L}=J / 2$. We first consider the microscopic index

$$
\widetilde{d}_{\text {micro }}\left(n, Q_{1}, Q_{5}, J\right) \equiv-\frac{1}{2 !} \widetilde{\operatorname{Tr}}\left[(-1)^{2 J_{3 R}}\left(2 J_{3 R}\right)^{2}\right]
$$

where the trace is taken over states carrying fixed $Q_{1}, Q_{5}, n$ and $J_{3 L}=J / 2$, but different values of $\vec{J}_{L}^{2}, J_{3 R}$ and $\vec{J}_{R}^{2}$. The expression for $\widetilde{d}_{\text {micro }}\left(n, Q_{1}, Q_{5}, J\right)$ can be obtained from the known expression for the elliptic genus of the D1-D5 conformal field theory [32]. It will however be convenient for us to begin with the expression for the index of quarter BPS states in the four dimensional theory obtained by compactifying type IIB string theory on $K 3 \times S^{1} \times \widetilde{S}^{1} / \mathbb{Z}_{\mathbf{N}}$ [10, 15, 18], and then use the fact that the latter is given by placing the five dimensional system we want in the background of a Kaluza-Klein monopole associated with the $\widetilde{S}^{1}$ compactification [95]. Thus we simply need to remove [84,96] from the index of the four dimensional black hole computed in [10,15, 18] the contribution of the Kaluza-Klein monopole, and the contribution from the supersymmetric quantum mechanics that binds the D1-D5 system to the KaluzaKlein monopole, and then multiply this by the contribution from some additional fermion zero modes which are present in the five dimensional system [91. This gives the microscopic index of such states to be (the $\mathbf{N}=1$ result was written down explicitly in [31])

$$
(-1)^{J} \widetilde{d}_{\text {micro }}\left(n, Q_{1}, Q_{5}, J\right)=-\frac{1}{\mathbf{N}} \int d \widetilde{\rho} \int d \widetilde{\sigma} \int d \widetilde{v} e^{-2 \pi i(\widetilde{\rho} n+\widetilde{\sigma} Q / \mathbf{N}+\widetilde{v} J)}\left(e^{\pi i \widetilde{v}}-e^{-\pi i \widetilde{v}}\right)^{4} \frac{f_{1}(\mathbf{N} \widetilde{\rho})}{\widetilde{\Phi}(\widetilde{\rho}, \widetilde{\sigma}, \widetilde{v})},
$$

where $\widetilde{\Phi}$ is a known function of its arguments [15-18] and can be found e.g. in eqs.(C.18) of the review [97,

$$
\begin{gathered}
f_{1}(\mathbf{N} \widetilde{\rho})=\eta(\widetilde{\rho})^{k+2} \eta(\mathbf{N} \widetilde{\rho})^{k+2}, \quad k+2 \equiv \frac{24}{\mathbf{N}+1}=\frac{1}{2}\left(n_{V}-3\right), \\
Q \equiv Q_{1} Q_{5} .
\end{gathered}
$$

The contour integration over the complex variables $(\widetilde{\rho}, \widetilde{\sigma}, \widetilde{v})$ in $(\mathbf{5 . 2})$ runs along the real axes in the range $(0,1),(0, \mathbf{N})$ and $(0,1)$ respectively at fixed values of $\operatorname{Im}(\widetilde{\rho}, \widetilde{\sigma}, \widetilde{v})$. The $(-1)^{J}$ factor on the left hand side of (5.2) can be traced to the fact that the index is normally defined with a $(-1)^{2 J_{3 L}+2 J_{3 R}}$ factor inserted into the trace whereas in the definition of $\widetilde{d}_{\text {micro }}$ we have just inserted $(-1)^{2 J_{3 R}}$ into the trace. $-1 / \widetilde{\Phi}$ is the partition function for the four dimensional index. On the other hand $1 / f_{1}(\mathbf{N} \widetilde{\rho})$ is the partition function of the index associated with the Kaluza-Klein monopole, and a factor of $-\left(e^{\pi i \widetilde{v}}-e^{-\pi i \widetilde{v}}\right)^{-2}$ represents the partition function associated with the supersymmetric quantum mechanics that describes the D1-D5 center of 
mass motion in the KK monopole background. Both these factors must be removed from the four dimensional partition function $-1 / \widetilde{\Phi}[84,96]$, accounting for a multiplicative factor of $-\left(e^{\pi i \widetilde{v}}-e^{-\pi i \widetilde{v}}\right)^{2} f_{1}(\mathbf{N} \widetilde{\rho})$ in the integrand. Another multiplicative factor of $-\left(e^{\pi i \widetilde{v}}-e^{-\pi i \widetilde{v}}\right)^{2}$ in (5.2) represents the index associated with the fermion zero modes of the D1-D5 system carrying non-trivial $S U(2)_{L}$ quantum numbers [31,91].

Using (4.14) for the microscopic index we can compute the index $d_{\text {micro }}\left(n, Q_{1}, Q_{5}, J\right)$ where we also fix $\vec{J}_{L}^{2}=\frac{J}{2}\left(\frac{J}{2}+1\right)$. This gives

$$
\begin{aligned}
& d_{\text {micro }}\left(n, Q_{1}, Q_{5}, J\right)=\widetilde{d}_{\text {micro }}\left(n, Q_{1}, Q_{5}, J\right)-\widetilde{d}_{\text {micro }}\left(n, Q_{1}, Q_{5}, J+2\right) \\
= & (-1)^{J+1} \frac{1}{\mathbf{N}} \int d \widetilde{\rho} \int d \widetilde{\sigma} \int d \widetilde{v} e^{-2 \pi i(\widetilde{\rho} n+\widetilde{\sigma} Q / \mathbf{N}+\widetilde{v} J)}\left(e^{\pi i \widetilde{v}}-e^{-\pi i \widetilde{v}}\right)^{4}\left(1-e^{-4 \pi i \widetilde{v}}\right) \frac{f_{1}(\mathbf{N} \widetilde{\rho})}{\widetilde{\Phi}(\widetilde{\rho}, \widetilde{\sigma}, \widetilde{v})}
\end{aligned}
$$

\subsection{Evaluation of the index}

(5.5) may be evaluated by deforming the contours of integration of $(\widetilde{\rho}, \widetilde{\sigma}, \widetilde{v})$ and picking up contributions from the residues at various poles. The leading contribution comes from the residue at [10,11, 15, 18] 6

$$
\widetilde{\rho} \widetilde{\sigma}-\widetilde{v}^{2}+\widetilde{v}=0
$$

where $\widetilde{\Phi}$ has a zero. We now make a change of variables

$$
\widetilde{\rho}=\frac{1}{\mathbf{N}} \frac{1}{2 v-\rho-\sigma}, \quad \widetilde{\sigma}=\mathbf{N} \frac{v^{2}-\rho \sigma}{2 v-\rho-\sigma}, \quad \widetilde{v}=\frac{v-\rho}{2 v-\rho-\sigma}
$$

or equivalently

$$
\rho=\frac{\widetilde{\rho \sigma}-\widetilde{v}^{2}}{\mathbf{N} \widetilde{\rho}}, \quad \sigma=\frac{\widetilde{\rho \sigma}-(\widetilde{v}-1)^{2}}{\mathbf{N} \widetilde{\rho}}, \quad v=\frac{\widetilde{\rho \sigma}-\widetilde{v}^{2}+\widetilde{v}}{\mathbf{N} \widetilde{\rho}}
$$

In these variables we have

$$
d \widetilde{\rho} \wedge d \widetilde{\sigma} \wedge d \widetilde{v}=-(2 v-\rho-\sigma)^{-3} d \rho \wedge d \sigma \wedge d v
$$

and the pole at (5.6) is situated at

$$
v=0 \text {. }
$$

\footnotetext{
${ }^{6}$ This was originally derived for the limit in which $Q_{1} Q_{5}$ and $n$ scale in the same way, and $J$ scales at either the same rate or slower than $Q_{1} Q_{5}$ and $n$. But a careful analysis shows that this pole also gives the dominant contribution in other scaling limits 31 .
} 
Furthermore $\widetilde{\Phi}$ satisfies (see e.g. eq.(C.21) of the review [97] where also all the other properties of $\widetilde{\Phi}$ and $\widehat{\Phi}$ discussed here can be found)

$$
\widetilde{\Phi}(\widetilde{\rho}, \widetilde{\sigma}, \widetilde{v})=-(i)^{k} C_{1}(2 v-\rho-\sigma)^{k} \widehat{\Phi}(\rho, \sigma, v)
$$

where $C_{1}$ is a real positive constant, $\widehat{\Phi}(\rho, \sigma, v)$ is a new function defined e.g in eq.(C.19) of [97], and $k$ has been defined in (5.3). Using these relations in (5.5) we get the leading contribution to $d_{\text {micro }}$ to be

$$
\begin{aligned}
d_{\text {micro }}\left(n, Q_{1}, Q_{5}, J\right) \simeq & (-1)^{J+1} \frac{(i)^{-k}}{\mathbf{N} C_{1}} \int_{\mathcal{C}^{\prime}} d \rho \wedge d \sigma \wedge d v(2 v-\rho-\sigma)^{-k-3} \frac{1}{\widehat{\Phi}(\rho, \sigma, v)} \\
& e^{-2 \pi i(\widetilde{\rho} n+\widetilde{\sigma} Q / \mathbf{N}+\widetilde{v} J)}\left(e^{\pi i \widetilde{v}}-e^{-\pi i \widetilde{v}}\right)^{4}\left(1-e^{-4 \pi \tau \widetilde{v}}\right) f_{1}(\mathbf{N} \widetilde{\rho})
\end{aligned}
$$

where $\mathcal{C}^{\prime}$ denotes a contour around $v=0$.

Now near $v=0 \widehat{\Phi}(\rho, \sigma, v)$ behaves as [15, 18, 97

$$
\widehat{\Phi}(\rho, \sigma, v)=-4 \pi^{2} v^{2} g(\rho) g(\sigma)+\mathcal{O}\left(v^{4}\right)
$$

where

$$
g(\rho)=\eta(\rho)^{k+2} \eta(\mathbf{N} \rho)^{k+2} .
$$

This allows us to evaluate the integration over $v$ in (5.12) using the residue theorem. Making a further change of variables

$$
\rho=\tau_{1}+i \tau_{2}, \quad \sigma=-\tau_{1}+i \tau_{2},
$$

so that near the pole $v=0$ we have

$$
\begin{aligned}
& \tilde{\rho}=\frac{i}{2 \mathbf{N} \tau_{2}}\left(1-i \frac{v}{\tau_{2}}+\mathcal{O}\left(v^{2}\right)\right), \quad \tilde{\sigma}=i \mathbf{N} \frac{\tau_{1}^{2}+\tau_{2}^{2}}{2 \tau_{2}}\left(1-i \frac{v}{\tau_{2}}+\mathcal{O}\left(v^{2}\right)\right), \\
& \widetilde{v}=\left(\frac{1}{2}-i \frac{\tau_{1}}{2 \tau_{2}}\right)\left(1-i \frac{v}{\tau_{2}}-\frac{v}{\tau_{1}+i \tau_{2}}+\mathcal{O}\left(v^{2}\right)\right),
\end{aligned}
$$

we can express (5.12) as

$$
d_{\text {micro }}\left(n, Q_{1}, Q_{5}, J\right) \simeq \int \frac{d^{2} \tau}{\tau_{2}^{2}} G\left(\tau_{1}, \tau_{2}\right)
$$

where

$$
G\left(\tau_{1}, \tau_{2}\right)=\exp \left[\frac{\pi}{\tau_{2}}\left\{\frac{n}{\mathbf{N}}+Q\left(\tau_{1}^{2}+\tau_{2}^{2}\right)-\tau_{1} J\right\}\right]\left\{g\left(\tau_{1}+i \tau_{2}\right) g\left(-\tau_{1}+i \tau_{2}\right)\right\}^{-1}\left(2 \tau_{2}\right)^{-\left(n_{V}-3\right) / 2}
$$




$$
\begin{aligned}
& \times f_{1}\left(\frac{i}{2 \tau_{2}}\right)\left\{2 \cosh \left(\frac{\pi \tau_{1}}{2 \tau_{2}}\right)\right\}^{4}\left(1-e^{-2 \pi \tau_{1} / \tau_{2}}\right) \\
& \times\left\{n_{V}-1+\frac{2 \pi}{\tau_{2}}\left(\frac{n}{\mathbf{N}}+Q\left(\tau_{1}^{2}+\tau_{2}^{2}\right)-\tau_{1} J\right)+i \frac{1}{\tau_{2}} \frac{f_{1}^{\prime}\left(i / 2 \tau_{2}\right)}{f_{1}\left(i / 2 \tau_{2}\right)}+4 \pi \frac{\tau_{1}}{\tau_{2}} \tanh \frac{\pi \tau_{1}}{2 \tau_{2}}\right. \\
& \left.+4 \pi \frac{\tau_{1}}{\tau_{2}} \frac{e^{-2 \pi \tau_{1} / \tau_{2}}}{1-e^{-2 \pi \tau_{1} / \tau_{2}}}\right\} \times \text { constant. }
\end{aligned}
$$

$\simeq$ in (5.17) implies equality up to exponentially suppressed contributions. If we had considered the index $\widetilde{d}_{\text {micro }}\left(n, Q_{1}, Q_{5}, J\right)$ then the factor of $\left(1-e^{-2 \pi \tau_{1} / \tau_{2}}\right)$ from the second line and the $4 \pi \frac{\tau_{1}}{\tau_{2}} \frac{e^{-2 \pi \tau_{1} / \tau_{2}}}{1-e^{-2 \pi \tau_{1} / \tau_{2}}}$ factor from the last line of (5.18) will be absent.

We now consider the limit $n \sim Q_{1} \sim Q_{5} \sim \Lambda, J^{2} \sim \Lambda^{3}$, with large $\Lambda$. In this case $Q \equiv Q_{1} Q_{5} \sim \Lambda^{2}$ and the leading contribution to (5.17) as well as systematic corrections to this formula can be found using saddle point method. The saddle point values of $\tau_{1}, \tau_{2}$ at the leading order, obtained by extremizing the exponent of the first exponential in (5.18) with respect to $\tau_{1}, \tau_{2}$, are given by

$$
\tau_{1}=\frac{J}{2 Q} \sim \Lambda^{-1 / 2}, \quad \tau_{2}=\sqrt{\frac{\frac{n}{\mathbf{N}}-\frac{J^{2}}{4 Q}}{Q}} \sim \Lambda^{-1 / 2} .
$$

Substituting this into the exponential of the first exponent in (5.18) we get the result $2 \pi \sqrt{Q_{1} Q_{5} \frac{n}{\mathbf{N}}-\frac{J^{2}}{4}}$ which is the leading contribution to the entropy given in (4.4).

In order to calculate the logarithmic corrections to the entropy we first note that the integration over the $\tau_{1}, \tau_{2}$ coordinates run along the imaginary $\tau_{1}, \tau_{2}$ directions [97]. In these directions the first exponential term in (5.18) is sharpely peaked around the saddle point with a width of order

$$
\Delta \tau_{1} \sim \Lambda^{-5 / 4}, \quad \Delta \tau_{2} \sim \Lambda^{-5 / 4}
$$

which can be found by studying the second derivative of the term in the exponent with respect to $\left(\tau_{1}, \tau_{2}\right)$. The logarithmic corrections to the log of the index come from the following factors in (5.17), (5.18) containing powers of $\Lambda$ :

$$
\begin{aligned}
\left(\tau_{2}\right)^{-2} & : \quad \Lambda \\
\Delta \tau_{1} & : \Lambda^{-5 / 4} \\
\Delta \tau_{2} & : \Lambda^{-5 / 4} \\
\{g(\tau) g(-\bar{\tau})\}^{-1}\left(2 \tau_{2}\right)^{-\left(n_{V}-3\right) / 2} & : \Lambda^{-\left(n_{V}-3\right) / 4} \\
\left\{n_{V}-1+\frac{2 \pi}{\tau_{2}}\left(\frac{n}{\mathbf{N}}+Q\left(\tau_{1}^{2}+\tau_{2}^{2}\right)-\tau_{1} J\right)+i \frac{1}{\tau_{2}} \frac{f_{1}^{\prime}\left(i / 2 \tau_{2}\right)}{f_{1}\left(i / 2 \tau_{2}\right)}+4 \pi \frac{\tau_{1}}{\tau_{2}} \tanh \frac{\pi \tau_{1}}{2 \tau_{2}}\right. &
\end{aligned}
$$




$$
\left.+4 \pi \frac{\tau_{1}}{\tau_{2}} \frac{e^{-2 \pi \tau_{1} / \tau_{2}}}{1-e^{-2 \pi \tau_{1} / \tau_{2}}}\right\}: \Lambda^{3 / 2}
$$

Note that in order to extract the small $\tau$ behaviour of $g(\tau)$, given in the fourth line of ([5.21), we need to make use of the modular properties of the $\eta$-functions appearing in (5.14). Multiplying the various factors in (5.21) we get a net contribution of

$$
\Lambda^{-\left(n_{V}-3\right) / 4}=\exp \left[-\frac{1}{4}\left(n_{V}-3\right) \ln \Lambda\right] .
$$

The term in the exponent on the right hand side of (5.22) is the net logarithmic correction to $\ln d_{\text {micro }}$. If instead of considering the index $d_{\text {micro }}$ we had analyzed $\widetilde{d}_{\text {micro }}$ we would have gotten an identical result since the extra factors mentioned below (5.18), which distinguish $d_{\text {micro }}$ from $\widetilde{d}_{\text {micro }}$, do not contribute to the logarithmic corrections. The result (15.22) for $d_{\text {micro }}$ is in perfect agreement with the macroscopic result (4.17).

We can also consider the case when $J \sim \Lambda^{(3 / 2)-\alpha}$ for some positive constant $\alpha$. In this case it follows from (5.19) that $\tau_{1} \sim \Lambda^{-(1 / 2)-\alpha}, \tau_{2} \sim \Lambda^{-1 / 2}$ and hence an additional logarithmic correction comes from the following factor in (5.18)

$$
\left(1-e^{-2 \pi \tau_{1} / \tau_{2}}\right) \quad: \quad \Lambda^{-\alpha}
$$

Thus we get the net power of $\Lambda$ in the expression for $d\left(n, Q_{1}, Q_{5}, J\right)$ to be

$$
\Lambda^{-\alpha-\left(n_{V}-3\right) / 4}=\exp \left[-\frac{1}{4}\left(n_{V}-3+4 \alpha\right) \ln \Lambda\right] \text {. }
$$

This agrees with the macroscopic result (6.2). If we had considered the index $\widetilde{d}_{\text {micro }}$ we would get the result (5.22) since the factor given in (5.23) is absent from the expression for $\widetilde{d}_{\text {micro }}$.

The above analysis needs some modification when $J$ vanishes exactly since the factor $\left(1-e^{-2 \pi \tau_{1} / \tau_{2}}\right)$ vanishes at the saddle point. In this case we proceed by expanding this in a power series in $\tau_{1}$ :

$$
\left(1-e^{-2 \pi \tau_{1} / \tau_{2}}\right)=2 \pi \frac{\tau_{1}}{\tau_{2}}-2 \pi^{2}\left(\frac{\tau_{1}}{\tau_{2}}\right)^{2}+\cdots
$$

Substituting this into (5.17), (5.18) we see that the contribution from the term linear in $\tau_{1}$ vanishes by $\tau_{1} \rightarrow-\tau_{1}$ symmetry of the rest of the integrand 7 The term proportional to $\left(\tau_{1}\right)^{2}$

\footnotetext{
${ }^{7}$ For this we need to ignore the last term inside the curly bracket in (5.18) which is in any case subdominant.
} 
can be evaluated by taking the $\tau_{1}$ integral to be approximately a gaussian with a width of order $\Delta \tau_{1} \sim \Lambda^{-5 / 4}$ around the saddle point. Thus $\left(\tau_{1}\right)^{2}$ factor can be replaced by a term of order $\Lambda^{-5 / 2}$. On the other hand since the saddle point value of $\tau_{2}$ is of order $\Lambda^{-1 / 2}$ which is larger than the width of the gaussian $\Lambda^{-5 / 4}$, we can replace the $\left(\tau_{2}\right)^{2}$ in the denominator by its saddle point value of order $\Lambda^{-1}$. This gives a net factor of $\Lambda^{-5 / 2} / \Lambda^{-1} \sim \Lambda^{-3 / 2}$, and we get the net logarithmic correction to $d_{\text {micro }}$ for $J=0$ to be

$$
\Lambda^{-3 / 2-\left(n_{V}-3\right) / 4}=\exp \left[-\frac{1}{4}\left(n_{V}+3\right) \ln \Lambda\right]
$$

in agreement with the macroscopic result (4.18). We now see that if we had used the index $\widetilde{d}_{\text {micro }}$ we would get the result (5.22) which will disagree with the macroscopic result. But as discussed in $\$ 4.2$ in this case $d_{\text {micro }}$ is the correct index to compare.

Before concluding this section we would like to mention that the results for the microscopic index exist for a more general class of $\mathcal{N}=4$ supersymmetric theories obtained by taking $\mathbb{Z}_{\mathbf{N}}$ orbifolds of type IIB string theory on $K 3 \times S^{1}$ with non-prime $\mathbf{N}$, as well as orbifolds of type IIB string theory on $T^{5}$. For these models the formula for the index takes a form similar to (5.2) and the relation between $k$ and $n_{V}$ given in (5.3) still holds although the relation between $\mathbf{N}$ and $n_{V}$ given in (5.3) is lost. Also the functions $g(\tau)$ and $f_{1}(\tau)$ have more complicated form, but their large and small $\tau$ behavior are identical to what we have discussed. Thus the results (5.22), (5.24) and (5.26) hold for these models as well.

\subsection{Removal of the additional hair contribution}

It has been argued in [91,98] that some hair degrees of freedom may live outside the horizon of the black hole and hence their contribution must be removed from the microscopic partition function before we can compare the results to the macroscopic index associated with the horizon degrees of freedom. The hair modes for a BMPV black hole in type IIB string theory on $K 3 \times S^{1}$ were analyzed in [98]. A similar analysis is also possible for the orbifold models. It was found in [98] that the hair mode contribution to the partition function includes a factor of $\left(e^{\pi i \widetilde{v}}-e^{-\pi i \widetilde{v}}\right)^{4}$ associated with the fermion zero modes carrying $J_{3 L}$ charge and also a $\widetilde{\rho}$ dependent factor $h(\widetilde{\rho})$ associated with the modes of the gravitino field. Thus we must multiply the integrand in (5.5) by a factor of $\left(e^{\pi i \widetilde{v}}-e^{-\pi i \widetilde{v}}\right)^{-4}(h(\widetilde{\rho}))^{-1}$. Now it follows from (5.16) that at the saddle point

$$
\left(e^{\pi i \widetilde{v}}-e^{-\pi i \widetilde{v}}\right)=i \cosh \frac{\pi \tau_{1}}{2 \tau_{2}}, \quad \widetilde{\rho}=\frac{i}{2 \mathrm{~N} \tau_{2}}
$$


Using (5.19) we see that $\left(e^{\pi i \widetilde{v}}-e^{-\pi i \widetilde{v}}\right)$ remains finite at the saddle point. On the other hand since $\widetilde{\rho} \sim i \Lambda^{1 / 2}$, and since $h(\widetilde{\rho})$ is made of products of $\left(1-e^{2 \pi i \ell \widetilde{\rho}}\right)[98], 8$ it does not give any factor involving powers of $\Lambda$. Thus removal of the hair contribution from the partition function does not introduce any new logarithmic correction to the entropy.

\subsection{BMPV black hole in type IIB on $T^{5}$}

We shall now briefly discuss the microscopic computation of the index in type IIB string theory on $T^{5}$. In this case the known microscopic index is [31, 37]

$$
\widetilde{d}_{\text {micro }}\left(n, Q_{1}, Q_{5}, J\right)=-\frac{1}{6 !} \widetilde{T r}\left[(-1)^{2 J_{3 R}}\left(2 J_{3 R}\right)^{6}\right],
$$

where, as before, we take the trace over states with fixed $Q_{1}, Q_{5}, n$ and $J_{3 L}=J / 2$, but all $J_{3 R}, \vec{J}_{R}^{2}$ and $\vec{J}_{L}^{2}$. Explicit computation gives this index to be 37]

$$
\widetilde{d}_{\text {micro }}\left(n, Q_{1}, Q_{5}, J\right) \simeq(-1)^{J} \int_{0}^{1} d \tau \int_{0}^{1} d v e^{-2 \pi i Q_{1} Q_{5} n \tau-2 \pi i J v}\left(e^{\pi i v}-e^{-\pi i v}\right)^{4} \frac{\vartheta_{1}(v \mid \tau)^{2}}{\eta(\tau)^{6}},
$$

up to exponentially suppressed terms. As in (5.5) we can find the index $d_{\text {micro }}$ for fixed $Q_{1}$, $Q_{5}, n, J_{3 R}=J / 2$ and $\vec{J}_{R}^{2}=\frac{J}{2}\left(\frac{J}{2}+1\right)$ by inserting in the integrand a factor of $\left(1-e^{-4 \pi i v}\right)$ :

$$
\begin{aligned}
& d_{\text {micro }}\left(n, Q_{1}, Q_{5}, J\right)=\widetilde{d}_{\text {micro }}\left(n, Q_{1}, Q_{5}, J\right)-\widetilde{d}_{\text {micro }}\left(n, Q_{1}, Q_{5}, J+2\right) \\
\simeq & (-1)^{J} \int_{0}^{1} d \tau \int_{0}^{1} d v e^{-2 \pi i Q_{1} Q_{5} n \tau-2 \pi i J v}\left(e^{\pi i v}-e^{-\pi i v}\right)^{4} \frac{\vartheta_{1}(v \mid \tau)^{2}}{\eta(\tau)^{6}}\left(1-e^{-4 \pi i v}\right) .
\end{aligned}
$$

We now consider the scaling limit:

$$
Q_{1}, Q_{5}, n \sim \Lambda, \quad J \sim \Lambda^{\frac{3}{2}-\alpha}
$$

and try to evaluate the integral using saddle point method. Anticipating that at the saddle point $\tau$ is small and $v \sim 1$, we can approximate the integral by

$$
\begin{aligned}
d_{\text {micro }}\left(n, Q_{1}, Q_{5}, J\right) \simeq(-1)^{J} \int_{0}^{1} d \tau & \int_{0}^{1} d v e^{-2 \pi i Q_{1} Q_{5} n \tau-2 \pi i J v}\left(e^{\pi i v}-e^{-\pi i v}\right)^{4} e^{-2 \pi i v^{2} / \tau} \\
& e^{2 \pi i v / \tau}\left(1-e^{-2 i \pi v / \tau}\right)^{2}(-i \tau)^{2}\left(1-e^{-4 \pi i v}\right)
\end{aligned}
$$

Extremizing the integrand with respect to $v$ and $\tau$ we find the approximate saddle point in the range $0 \leq \operatorname{Re}(v)<1$ at 31 ]

$$
v=\frac{1}{2}-\frac{J}{2} \tau+\cdots, \quad \tau=i / \sqrt{4 n Q_{1} Q_{5}-J^{2}}+\cdots \sim \Lambda^{-3 / 2},
$$

\footnotetext{
${ }^{8}$ For type IIB on $K 3 \times S^{1}$ we have $h(\widetilde{\rho})=\prod_{\ell=1}^{\infty}\left(1-e^{2 \pi i \ell \widetilde{\rho}}\right)^{4}$.
} 
where $\cdots$ denote subleading terms. The value of the integrand at this saddle point is

$$
\exp \left[\pi \sqrt{4 n Q_{1} Q_{5}-J^{2}}+\cdots\right]
$$

This gives the leading contribution to $d_{\text {micro }}\left(n, Q_{1}, Q_{5}, J\right)$. To examine the logarithmic corrections we determine the various powers of $\Lambda$ coming from different terms in (5.32):

$$
\begin{aligned}
\Delta v & : \Lambda^{-3 / 4} \\
\Delta \tau & : \Lambda^{-9 / 4} \\
(-i \tau)^{2} & : \Lambda^{-3} \\
\left(1-e^{-4 \pi i v}\right) & : \Lambda^{-\alpha}
\end{aligned}
$$

where $\Delta v$ and $\Delta \tau$ denotes the range of $v$ and $\tau$ integration beyond which the integrand falls off sharply. Taking the product of these factors we get the net power of $\Lambda$ in the expression for the index:

$$
\Lambda^{-6-\alpha}=\exp [-(6+\alpha) \ln \Lambda]
$$

For $\alpha=0$ this agrees with the macroscopic result (4.17) for $n_{V}=27$. On the other hand for $\alpha>0$, (5.36) agrees with the macroscopic result (6.2). For computing the index $\widetilde{d}_{\text {micro }}$ we need to drop the $\left(1-e^{-4 \pi i v}\right)$ from the integrand in (5.32) with no other change. According to (5.35), we now get the result

$$
\Lambda^{-6}=\exp [-6 \ln \Lambda]
$$

If we instead set $J=0$ then the relevant index for comparison with the macroscopic result $d_{\text {micro. }}$ In this case special care is needed to deal with the factor of $\left(1-e^{-4 \pi i v}\right)$. Since this vanishes at the saddle point, we expand it to second order in fluctuations about the saddle point and replace it by a term of order $\Delta v^{2} \sim \Lambda^{-3 / 2}$. This gives a net contribution of

$$
\Lambda^{-6-3 / 2}=\exp \left[-\frac{15}{2} \ln \Lambda\right]
$$

to $d_{\text {micro. }}$. Again this agrees with the macroscopic result (4.18) for $n_{V}=27$. However if we had considered the index $\widetilde{d}_{\text {micro }}$ we would have gotten the result (5.37) which would disagree with the macroscopic result. This again illustrates the necessity for making the correct choice of ensembles while comparing the microscpic and the macroscopic results. 


\subsection{Comparison with the Cardy limit}

Since the success of the black hole microstate counting is often associated with the Cardy formula - applicable for $n \rightarrow \infty$ limit at fixed $Q_{1}, Q_{5}$ - we shall analyze in this subsection the logarithmic correction to the entropy in the Cardy limit. For this we consider the scaling

$$
n \sim \Lambda^{3}, \quad J \sim \Lambda^{3 / 2-\alpha}
$$

with $Q_{1}, Q_{5}$ fixed. Eq.(5.19) shows that in this limit

$$
\tau_{2} \sim \Lambda^{3 / 2}, \quad \tau_{1} \sim \Lambda^{3 / 2-\alpha}
$$

Thus the logarithmic correction to $\ln d_{\text {micro }}$ comes from the terms:

$$
\begin{aligned}
\left(\tau_{2}\right)^{-2} & : \Lambda^{-3} \\
\Delta \tau_{1} & : \Lambda^{3 / 4} \\
\Delta \tau_{2} & : \Lambda^{3 / 4} \\
g(\tau) g(-\bar{\tau})\left(2 \tau_{2}\right)^{-\left(n_{V}-3\right) / 2} & : \Lambda^{-3\left(n_{V}-3\right) / 4} \\
f_{1}\left(i / 2 \tau_{2}\right) & : \Lambda^{3\left(n_{V}-3\right) / 4} \\
\left\{n_{V}-1+\frac{2 \pi}{\tau_{2}}\left(\frac{n}{\mathbf{N}}+Q\left(\tau_{1}^{2}+\tau_{2}^{2}\right)-\tau_{1} J\right)+i \frac{1}{\tau_{2}} \frac{f_{1}^{\prime}\left(i / 2 \tau_{2}\right)}{f_{1}\left(i / 2 \tau_{2}\right)}+4 \pi \frac{\tau_{1}}{\tau_{2}} \tanh \frac{\pi \tau_{1}}{2 \tau_{2}}\right. & \\
+4 \pi \frac{\tau_{1}}{\tau_{2}} \frac{e^{-2 \pi \tau_{1} / \tau_{2}}}{\left.1-e^{-2 \pi \tau_{1} / \tau_{2}}\right\}} & : \Lambda^{3 / 2} \\
\left(1-e^{-2 \pi \tau_{1} / \tau_{2}}\right) & : \quad \Lambda^{-\alpha}
\end{aligned}
$$

Note that we now need to use the modular property of the $\eta$-functions appearing in (5.3) to find the behavior of $f_{1}$ for small value of its argument. Taking the product of all the factors we get 9

$$
\Lambda^{-\alpha}=\exp [-\alpha \ln \Lambda]
$$

which is quite different from (5.24). In particular there is no dependence on $n_{V}$. This shows that the Cardy limit is not always reliable for extracting the black hole entropy in the limit

\footnotetext{
${ }^{9}$ If we had been considering the elliptic genus of symmetric products of $\mathrm{K} 3$ - which is a weak Jacobi form of weight zero - then for $\alpha=0$ we should have gotten a power correction of order $n^{-1} \sim \Lambda^{-3} 38$. However the contribution from the center of mass oscillation modes turns the elliptic genus into a (meromorphic) weak Jacobi form of weight two, and kills the logarithmic term in the asymptotic expansion of the Fourier coefficients.
} 
where the supergravity approximation is reliable. Similar observations have been made earlier in [31,84], and it has in fact been argued in [31] that the limit $Q_{1} Q_{5}>>n$ is related to the Cardy limit of a different CFT that appears in the dual type IIA description.

We can also consider a similar limit for the formula (5.30) for type IIB string theory on $T^{5}$. From (5.30) we see that the index depends only on the combination $Q_{1} Q_{5} n$ and not individually on $Q_{1}, Q_{5}$ and $n$. Thus in this case scaling $n$ by $\Lambda^{3}$ keeping $Q_{1}$ and $Q_{5}$ fixed is equivalent to scaling $Q_{1}, Q_{5}$ and $n$ by $\Lambda$, and we get a result identical to (5.36):

$$
\exp [-(6+\alpha) \ln \Lambda]
$$

\section{Slowly rotating black holes}

In the macroscopic analysis of $\$ 4$ we have considered two kinds of cases - $(i)$ when the angular momentum is large so that its contribution to the classical Bekenstein-Hawking entropy scales in the same way as that from the charges, and (ii) when the angular momentum vanishes. In this section we shall consider some cases when the angular momentum is non-zero but parametrically small so that it gives negligible contribution to the classical Bekenstein-Hawking entropy. This analyis will however require us to make some assumptions about the spectrum of the kinetic operator in the near horizon geometry of a slowly rotating black hole. For this reason the results of this section should be regarded as somewhat tentative.

\subsection{Slowly rotating BMPV black holes}

The microscopic analysis for BMPV black holes yields the result for the index in the scaling limit $Q_{1}, Q_{5}, n \sim \Lambda, J \sim \Lambda^{\frac{3}{2}-\alpha}$ for some positive number $\alpha$ - as given in eqs.(5.24) and (5.36). Since $J / \Lambda^{3 / 2}$ is the parameter that controls the deviation of the black hole solution from the rotationally invariant configuration, our first guess will be that the partition function on the macroscopic side can be computed using perturbation expansion in $J / \Lambda^{3 / 2}$ around the rotationally invariant configuration $J=0$. Furthermore, one would naively expect that the corrections to the non-zero eigenvalues would have negligible effect for small $J / \Lambda^{\frac{3}{2}}$ and we only need to take into account the shift in the zero eigenvalues using perturbation theory. This would produce small but non-zero eigenvalues, and, as a result, compared to the case of $J \sim \Lambda^{3 / 2}$, the one loop determinant will get some extra power of $J / \Lambda^{\frac{3}{2}}$ from the small eigenvalues. However this naive expectation is not quite correct due to the following reason. We recall that the zero 
modes which are lifted by switching on $J$ are the ones associated with the $W_{L}^{ \pm}$gauge fields on $A d S_{2}$ arising from $S U(2)_{L}$. Now a non-zero $J$ corresponds to switching on a constant $U(1)_{L}$ electric field along $A d S_{2}$. Since the $W_{L}^{ \pm}$fields are charged under the $U(1)_{L}$, any quantum of $W_{L}^{ \pm}$will be subject to a constant force in the presence of a $U(1)_{L}$ electric field. Hence we cannot use perturbation theory to study the effect of the switching on a $U(1)_{L}$ electric field on the zero modes of $W_{L}^{ \pm}$- we expect these zero modes to be lifted altogether in the presence of non-zero $J 10$ While we do not have a concrete analysis of the eigenvalue equations in the new background, it is natural to assume that all the modes, except the exact zero modes which are in any case neutral under $U(1)_{L}$, will have eigenvalues of order $a^{-2}$. In other words the effect of switching on even a small $J$ takes us to back to the case of generic $J / \Lambda^{3 / 2} \sim 1$, 1.e. gives a logarithmic correction to $\ln \widetilde{d}_{\text {macro }}\left(n, Q_{1}, Q_{5}, J\right)$ given in (4.16). Using the fact that $S_{B H}$ given in (4.4) satisfies

$$
S_{B H}(J)-S_{B H}(J+2) \simeq \frac{\pi}{2} \frac{J}{\sqrt{Q_{1} Q_{5} \widetilde{n}}} \sim \Lambda^{-\alpha}
$$

for $J \sim \Lambda^{\frac{3}{2}-\alpha}$, we see from (4.17) that the logarithmic correction to $d_{\text {macro }}\left(n, Q_{1}, Q_{5}, J\right)$ now takes the form:

$d_{\text {macro }}\left(n, Q_{1}, Q_{5}, J\right)=e^{S_{B H}(J)-\frac{1}{4}\left(n_{V}-3\right) \ln \Lambda+\cdots}-e^{S_{B H}(J+2)-\frac{1}{4}\left(n_{V}-3\right) \ln \Lambda+\cdots}=e^{S_{B H}(J)-\frac{1}{4}\left(n_{V}-3+4 \alpha\right) \ln \Lambda+\cdots}$.

This agrees with the microscopic result (5.24) and also with (5.36) for $n_{V}=27$.

\subsection{Slowly rotating extremal Kerr-Newmann black hole in four di- mensions}

In this subsection we shall briefly discuss the case of slowly rotating extremal Kerr-Newmann black hole in four dimensional Einstein-Maxwell theory. For zero angular momentum, 1.e. for Reissner-Nordstrom black hole, the result for the logarithmic correction was calculated in [27] with the result:

$$
-\frac{1}{180}\left(964+n_{S}+62 n_{V}+11 n_{F}\right) \ln A_{H}
$$

if the theory contains, besides the metric and the Maxwell field under which the black hole is charged, $n_{S}$ minimally coupled massless scalar fields, $n_{V}$ minimally coupled additional massless

\footnotetext{
${ }^{10}$ This is corroborated by the fact that if in $\mathrm{A.2}$ we replace $d \Phi^{(\ell)}$ by $D \Phi^{(\ell)}$ where $D$ denotes the gauge covariant derivative for the $W_{L}^{ \pm}$fields in the background of $U(1)_{L}$ electric field, then the modes cease to be square integrable.
} 
vector fields and $n_{F}$ minimally coupled massless Dirac fermions. Here $A_{H} \sim a^{2}$ is the area of the event horizon and we have set the Newton's constant $G_{N}$ to unity.

Let us now consider the effect of deforming the solution so that the black hole carries not only electric charge $q$ but also angular momentum $J$ while remaining extremal. The near horizon geometry still contains an $A d S_{2}$ factor [61, 62], but the $S O(3)$ isometry of the near horizon geometry is now broken to $U(1)$ and as a result from the dimensional reduction of the metric we get only one massless gauge field on $A d S_{2}$ instead of three. The departure of the metric from that of the Reissner-Nordstrom black hole is given by the rotation parameter

$$
\gamma \equiv \frac{J}{q^{2}}
$$

We shall work with $\gamma$ small (but still $J$ large) so that the geometry is almost that of the extremal Reissner-Nordstrom black hole. Following the discussion in $\$ 6.1$ we shall assume that the effect of switching on even a small $\gamma$ will be to get rid of the zero modes of two of the three gauge fields coming from the unbroken $S O(3)$ isometry of the non-rotating black holes and make all the eigenvalues, except those associated with the exact zero modes, of order $a^{-2}$. In the language of this paper, this translates to the fact that $2 \pi \int d^{2} y G(y) \bar{K}^{m}(y)$, instead of taking the value $3+3=6$ will now take value $1+3=4$. Since here $\beta_{m}=2$, this means according to (2.22) that we lose an additive contribution of $-2 \ln a=-\ln A_{H}$ from the entropy. Adding $\ln A_{H}$ to (6.3) we get

$$
\widetilde{d}_{\text {macro }}(q, J)=\exp \left[S_{B H}(q, J)-\frac{1}{180}\left(784+n_{S}+62 n_{V}+11 n_{F}\right) \ln A_{H}+\cdots\right] .
$$

Note that we have used the fact that for non-zero angular momentum the macroscopic computation yields the degeneracy $\widetilde{d}_{\text {macro }}(q, J)$ in the ensemble of fixed $J_{3}=J$, instead of $d_{\text {macro }}(q, J)$ which will be the degeneracy in the ensemble of fixed $J_{3}=J$ and $\vec{J}^{2}=J(J+1)$. However we can use (6.5) and the result for the classical Bekenstein-Hawking entropy:

$$
S_{B H}(q, J)=2 \pi \sqrt{q^{4}+J^{2}}=2 \pi q^{2}\left(1+\frac{1}{2} \gamma^{2}+\mathcal{O}\left(\gamma^{4}\right)\right)
$$

to compute $d_{\text {macro }}(q, J)$ :

$$
\begin{aligned}
d_{\text {macro }}(q, J) & =\widetilde{d}_{\text {macro }}(q, J)-\widetilde{d}_{\text {macro }}(q, J+1) \\
& \simeq \exp \left[S_{B H}(q, J)-\frac{1}{180}\left(784+n_{S}+62 n_{V}+11 n_{F}\right) \ln A_{H}+\ln \gamma\right]
\end{aligned}
$$


Acknowledgement: I wish to thank Monica Guica and Rajesh Gupta for useful communications. This work was supported in part by the J. C. Bose fellowship of the Department of Science and Technology, India and the project 11-R\&D-HRI-5.02-0304.

\section{A Counting of zero modes in $A d S_{2}$}

In this appendix we shall review the results on the zero modes of various fields in $A d S_{2}$ and count their numbers. For definiteness we shall take the $A d S_{2}$ metric to be

$$
\left(g_{A d S_{2}}\right)_{\mu \nu} d x^{\mu} d x^{\nu}=a^{2}\left(d \eta^{2}+\sinh ^{2} \eta d \theta^{2}\right),
$$

although the result for the number of zero modes will be independent of what we take to be the $A d S_{2}$ size $a$.

First consider the case of a $U(1)$ gauge field $A_{\mu}$. The normalized basis of zero modes of such a field on $A d S_{2}$ is given by 99

$$
A=d \Phi^{(\ell)}, \quad \Phi^{(\ell)}=\frac{1}{\sqrt{2 \pi|\ell|}}\left[\frac{\sinh \eta}{1+\cosh \eta}\right]^{|\ell|} e^{i \ell \theta}, \quad \ell= \pm 1, \pm 2, \pm 3, \cdots
$$

satisfying

$$
\int_{A d S_{2}} d^{2} x \sqrt{g_{A d S_{2}}} g_{A d S_{2}}^{m n} \partial_{m} \Phi^{(\ell) *} \partial_{n} \Phi^{\left(\ell^{\prime}\right)}=\delta_{\ell \ell^{\prime}}
$$

The basis states (A.2) also satisfy

$$
\sum_{\ell} g_{A d S_{2}}^{m n} \partial_{m} \Phi^{(\ell) *}(x) \partial_{n} \Phi^{(\ell)}(x)=\frac{1}{2 \pi a^{2}} .
$$

This can be derived using the fact that due to homogeneity of $A d S_{2}$ this sum is independent of $x$ and hence can be evaluated at $\eta=0$, and that at $\eta=0$ only the $\ell= \pm 1$ terms contribute to the sum. Thus the total number of such zero modes of a vector field on $A d S_{2}$ is given by

$$
N_{1}=\int_{A d S_{2}} d^{2} x \sqrt{g_{A d S_{2}}} \sum_{\ell} g_{A d S_{2}}^{m n} \partial_{m} \Phi^{(\ell) *}(x) \partial_{n} \Phi^{(\ell)}(x)=\frac{1}{2 \pi} \int_{0}^{\eta_{0}} \sinh \eta d \eta \int d \theta=\cosh \eta_{0}-1 .
$$

A similar analysis can be done for a symmetric rank two tensor representing the graviton fluctuation on $A d S_{2}$. The normalized basis of zero mode deformations is given by [99]

$$
\begin{aligned}
& h_{m n}=w_{m n}^{(\ell)}, \\
& w_{m n}^{(\ell)} d x^{m} d x^{n}=\frac{a}{\sqrt{\pi}}\left[\frac{|\ell|\left(\ell^{2}-1\right)}{2}\right]^{1 / 2} \frac{(\sinh \eta)^{|\ell|-2}}{(1+\cosh \eta)^{|\ell|}} e^{i \ell \theta}\left(d \eta^{2}+2 i \sinh \eta d \eta d \theta-\sinh ^{2} \eta d \theta^{2}\right) \\
& \ell \in \mathbb{Z}, \quad|\ell| \geq 2 .
\end{aligned}
$$


Locally these can be regarded as deformations generated by a diffeomorphism on $A d S_{2}$, but these diffeomorphisms themselves are not square integrable. The basis states (A.6) satisfy

$$
\sum_{\ell} g_{A d S_{2}}^{m n} g_{A d S_{2}}^{p q} w_{m p}^{(\ell) *}(x) w_{n q}^{(\ell)}(x)=\frac{3}{2 \pi a^{2}} .
$$

We have derived this using the fact that due to homogeneity of $A d S_{2}$ this sum is independent of $x$, and that at $\eta=0$ only the $\ell= \pm 2$ terms contribute to the sum. Thus as in (A.5) the total number of such discrete modes is given by

$$
N_{2}=3 \cosh \eta_{0}-3
$$

Finally we turn to the zero modes of the gravitino fields. We use the following conventions for the zweibeins and the gamma matrices

$$
\begin{gathered}
e^{0}=a \sinh \eta d \theta, \quad e^{1}=a d \eta \\
\gamma^{0}=-\tau_{2}, \quad \gamma^{1}=\tau_{1}
\end{gathered}
$$

where $\tau_{i}$ are two dimensional Pauli matrices and $a$ is the $A d S_{2}$ size parameter. In this convention the Dirac operator on $A d S_{2}$ can be written as

$$
\not D_{A d S_{2}}=a^{-1}\left[-\tau^{2} \frac{1}{\sinh \eta} \partial_{\theta}+\tau^{1} \partial_{\eta}+\frac{1}{2} \tau^{1} \operatorname{coth} \eta\right] .
$$

The eigenstates of $\not D_{A d S_{2}}$ are given by [100]

$$
\begin{aligned}
& \chi_{k}^{ \pm}(\lambda)= \frac{1}{\sqrt{4 \pi a^{2}}}\left|\frac{\Gamma(1+k+i \lambda)}{\Gamma(k+1) \Gamma\left(\frac{1}{2}+i \lambda\right)}\right| e^{i\left(k+\frac{1}{2}\right) \theta} \\
&\left(\begin{array}{c}
i \frac{\lambda}{k+1} \cosh ^{k} \frac{\eta}{2} \sinh ^{k+1} \frac{\eta}{2} F\left(k+1+i \lambda, k+1-i \lambda ; k+2 ;-\sinh ^{2} \frac{\eta}{2}\right) \\
\pm \cosh ^{k+1} \frac{\eta}{2} \sinh ^{k} \frac{\eta}{2} F\left(k+1+i \lambda, k+1-i \lambda ; k+1 ;-\sinh ^{2} \frac{\eta}{2}\right)
\end{array}\right), \\
& \eta_{k}^{ \pm}(\lambda)=\frac{1}{\sqrt{4 \pi a^{2}}}\left|\begin{array}{c}
\Gamma(1+k+i \lambda) \\
\Gamma(k+1) \Gamma\left(\frac{1}{2}+i \lambda\right)
\end{array}\right| e^{-i\left(k+\frac{1}{2}\right) \theta} \\
& \quad\left(\begin{array}{c}
\cosh ^{k+1} \frac{\eta}{2} \sinh ^{k} \frac{\eta}{2} F\left(k+1+i \lambda, k+1-i \lambda ; k+1 ;-\sinh ^{2} \frac{\eta}{2}\right) \\
\pm i \frac{\lambda}{k+1} \cosh ^{k} \frac{\eta}{2} \sinh ^{k+1} \frac{\eta}{2} F\left(k+1+i \lambda, k+1-i \lambda ; k+2 ;-\sinh ^{2} \frac{\eta}{2}\right)
\end{array}\right), \\
& k \in \mathbb{Z}, \quad 0 \leq k<\infty, \quad 0<\lambda<\infty,
\end{aligned}
$$


satisfying

$$
\not D_{A d S_{2}} \chi_{k}^{ \pm}(\lambda)= \pm i a^{-1} \lambda \chi_{k}^{ \pm}(\lambda), \quad \not D_{A d S_{2}} \eta_{k}^{ \pm}(\lambda)= \pm i a^{-1} \lambda \eta_{k}^{ \pm}(\lambda)
$$

The zero modes of the gravitino fields $\psi_{m}$ can be expressed in terms of the spinors (A.12) via the relations

$$
\xi_{m}^{(k) \pm} \equiv \mathcal{N}_{k}^{ \pm}\left(D_{m} \pm \frac{1}{2 a} \gamma_{m}\right) \chi_{k}^{ \pm}(i), \quad \widehat{\xi}_{m}^{(k) \pm} \equiv \widehat{\mathcal{N}}_{k}^{ \pm}\left(D_{m} \pm \frac{1}{2 a} \gamma_{m}\right) \eta_{k}^{ \pm}(i), \quad k=1, \cdots \infty
$$

where $\mathcal{N}_{k}^{ \pm}$and $\widehat{\mathcal{N}}_{k}^{ \pm}$are appropriate normalization constants such that

$$
a^{2} \int \sinh \eta d \eta d \theta g_{A d S_{2}}^{m n} \xi_{m}^{(k)+\dagger}(\eta, \theta) \xi_{n}^{\left(k^{\prime}\right)+}(\eta, \theta)=\delta_{k k^{\prime}}
$$

etc. Although $\chi_{k}^{ \pm}(i)$ and $\eta_{k}^{ \pm}(i)$ are not square integrable, the modes described in (A.14) are square integrable and hence they must be included among the eigenstates of the RaritaSchwinger operator. These modes can be shown to satisfy the chirality projection condition

$$
\tau_{3} \xi_{m}^{(k) \pm}=-\xi_{m}^{(k) \pm}, \quad \tau_{3} \widehat{\xi}_{m}^{(k) \pm}=\widehat{\xi}_{m}^{(k) \pm}
$$

Furthermore with the help of $(\underline{\mathrm{A} .13})$ and that $\chi_{k}^{-}=\tau_{3} \chi_{k}^{+}, \eta_{k}^{-}=\tau_{3} \eta_{k}^{+}$one can show that $\xi_{m}^{(k) \pm}$ are proportional to each other and $\widehat{\xi}_{m}^{(k) \pm}$ are proportional to each other.

Now suppose we have a set of gravitino zero modes given by $\xi_{m}^{(k)+}$ with $k$ ranging over all positive integers. Then the total number of zero modes may be expressed as

$$
\sum_{k=1}^{\infty} 1=a^{2} \int \sinh \eta d \eta d \theta \sum_{k=1}^{\infty} g^{m n} \xi_{m}^{(k)+\dagger}(\eta, \theta) \xi_{n}^{(k)+}(\eta, \theta) .
$$

We now use the fact that after taking the sum over $k$ the integrand must become independent of $(\eta, \theta)$ and hence we can evaluate it at $\eta=0$. In this case the only contribution comes from the $k=1$ term. Substituting $k=1, \lambda=i$ in (A.12) and choosing the normalization constant $\mathcal{N}_{1}^{ \pm}$so that $\xi_{m}^{(1)+}$ defined in (A.14) is normalized, we get

$$
\xi_{\theta}^{(1)+}=\frac{1}{\sqrt{\pi}} e^{3 i \theta / 2}\left(\begin{array}{c}
0 \\
\sinh \frac{\eta}{2} / \cosh ^{2} \frac{\eta}{2}
\end{array}\right), \quad \xi_{\eta}^{(1)+}=-\frac{i}{\sinh \eta} \xi_{\theta}^{(1)+} .
$$

This gives

$$
\sum_{k=1}^{\infty} g^{m n} \xi_{m}^{(k)+\dagger}(\eta, \theta) \xi_{n}^{(k)+}(\eta, \theta)=\left.g^{m n} \xi_{m}^{(1)+\dagger}(\eta, \theta) \xi_{n}^{(1)+}(\eta, \theta)\right|_{\eta=0}=\frac{1}{2 \pi a^{2}}
$$


Substituting this into (A.17) we get the total number of zero modes to be $\left(\cosh \eta_{0}-1\right)$. A similar contribution is found from the gravitino zero modes given by $\widehat{\xi}_{m}^{(k)+}$.

We should however remember that the spinors $\xi_{m}^{(k)+}$ and $\widehat{\xi}_{m}^{(k)+}$ are tensored with spinors associated with the tangent space spinors of other directions transverse to $A d S_{2}$ and hence each zero mode associated with $\xi_{m}^{(k)+}$ and $\widehat{\xi}_{m}^{(k)+}$ may actually represent multiple zero modes. In order to determine this multiplicity we shall use the fact that the gravitino zero modes are associated with the deformations generated by the fermionic generators of the $\mathcal{N}=4$ superconformal algebra labelled as $G_{n}^{\alpha \beta}$ where $\alpha$ and $\beta$ each takes value \pm 1 and $n \in \mathbb{Z}+\frac{1}{2}[30,67] . G_{ \pm 1 / 2}^{\alpha \beta}$ are the global symmetry generators which, together with the bosonic generators, form the $S U(1,1 \mid 2)$ supersymmetry of the near horizon geometry [67,68, 101]. In the present context

$G_{n}^{\alpha \beta}$ for $n \geq \frac{3}{2}$ can be identified with the zero modes $\xi_{m}^{(k)+}$ with $n=k+\frac{1}{2}$ and $G_{n}^{\alpha \beta}$ for $n \leq-\frac{3}{2}$ can be identified with the zero modes $\widehat{\xi}_{m}^{(k)+}$ with $|n|=k+\frac{1}{2}$. Since for each $n$ there are four generators labelled by the pair $(\alpha, \beta)$ we see that the zero modes associated with $\xi_{m}^{(k)+}$ and $\widehat{\xi}_{m}^{(k)+}$ must each have multiplicity 4 . This gives the total number of fermion zero modes to be

$$
N_{3 / 2}=8\left(\cosh \eta_{0}-1\right)
$$

Explicit construction of the four zero modes for each $\xi_{m}^{(k)+}$ (and $\widehat{\xi}_{m}^{(k)+}$ ) can be found in [26, 27]) for BPS black holes in four dimensional $\mathcal{N}=2$ and $\mathcal{N}=4$ supersymmetric theories.

\section{References}

[1] A. Strominger and C. Vafa, "Microscopic Origin of the Bekenstein-Hawking Entropy," Phys. Lett. B 379, 99 (1996) arXiv:hep-th/9601029.

[2] J. C. Breckenridge, R. C. Myers, A. W. Peet and C. Vafa, "D-branes and spinning black holes," Phys. Lett. B 391, 93 (1997) arXiv:hep-th/9602065.

[3] R. M. Wald, "Black hole entropy in the Noether charge," Phys. Rev. D 48, 3427 (1993) arXiv:gr-qc/9307038.

[4] T. Jacobson, G. Kang and R. C. Myers, "On Black Hole Entropy," Phys. Rev. D 49, 6587 (1994) arXiv:gr-qc/9312023].

[5] V. Iyer and R. M. Wald, "Some properties of Noether charge and a proposal for dynamical black hole entropy," Phys. Rev. D 50, 846 (1994) arXiv:gr-qc/9403028.

[6] T. Jacobson, G. Kang and R. C. Myers, "Black hole entropy in higher curvature gravity," arXiv:gr-qc/9502009.

[7] G. Lopes Cardoso, B. de Wit and T. Mohaupt, "Corrections to macroscopic supersymmetric black-hole entropy," Phys. Lett. B 451, 309 (1999) arXiv:hep-th/9812082. 
[8] G. Lopes Cardoso, B. de Wit and T. Mohaupt, "Deviations from the area law for supersymmetric black holes," Fortsch. Phys. 48, 49 (2000) arXiv:hep-th/9904005.

[9] T. Mohaupt, "Black hole entropy, special geometry and strings," Fortsch. Phys. 49, 3 (2001) [arXiv:hep-th/0007195].

[10] R. Dijkgraaf, E. P. Verlinde and H. L. Verlinde, "Counting dyons in N = 4 string theory," Nucl. Phys. B 484, 543 (1997) [arXiv:hep-th/9607026].

[11] G. Lopes Cardoso, B. de Wit, J. Kappeli and T. Mohaupt, "Asymptotic degeneracy of dyonic N = 4 string states and black hole entropy," JHEP 0412, 075 (2004) arXiv:hep-th/0412287.

[12] D. Shih, A. Strominger and X. Yin, "Recounting dyons in N = 4 string theory," arXiv:hep-th/0505094.

[13] D. Gaiotto, "Re-recounting dyons in N = 4 string theory," arXiv:hep-th/0506249

[14] D. Shih and X. Yin, "Exact black hole degeneracies and the topological string," JHEP 0604, 034 (2006) arXiv:hep-th/0508174.

[15] D. P. Jatkar and A. Sen, "Dyon spectrum in CHL models," JHEP 0604, 018 (2006) [arXiv:hep-th/0510147].

[16] J. R. David, D. P. Jatkar and A. Sen, "Product representation of dyon partition function in CHL models," JHEP 0606, 064 (2006) arXiv:hep-th/0602254.

[17] A. Dabholkar and S. Nampuri, "Spectrum of dyons and black holes in CHL orbifolds using Borcherds lift," arXiv:hep-th/0603066.

[18] J. R. David and A. Sen, "CHL dyons and statistical entropy function from D1-D5 system," arXiv:hep-th/0605210.

[19] J. R. David, D. P. Jatkar and A. Sen, "Dyon spectrum in N = 4 supersymmetric type II string theories," arXiv:hep-th/0607155.

[20] J. R. David, D. P. Jatkar and A. Sen, "Dyon spectrum in generic N = 4 supersymmetric Z(N) orbifolds," arXiv:hep-th/0609109.

[21] A. Strominger, "Black hole entropy from near-horizon microstates," JHEP 9802, 009 (1998) arXiv:hep-th/9712251].

[22] M. Guica, T. Hartman, W. Song, A. Strominger, "The Kerr/CFT Correspondence," Phys. Rev. D80, 124008 (2009). arXiv:0809.4266 [hep-th]].

[23] A. Sen, "Quantum Entropy Function from $\operatorname{AdS}(2) / C F T(1)$ Correspondence," Int. J. Mod. Phys. A 24, 4225 (2009) [arXiv:0809.3304 [hep-th]].

[24] I. Mandal, A. Sen, "Black Hole Microstate Counting and its Macroscopic Counterpart," Class. Quant. Grav. 27, 214003 (2010). arXiv:1008.3801 [hep-th]].

[25] S. Banerjee, R. K. Gupta, A. Sen, "Logarithmic Corrections to Extremal Black Hole Entropy from Quantum Entropy Function," JHEP 1103, 147 (2011) arXiv:1005.3044 [hep-th]].

[26] S. Banerjee, R. K. Gupta, I. Mandal, A. Sen, "Logarithmic Corrections to N=4 and N=8 Black Hole Entropy: A One Loop Test of Quantum Gravity," arXiv:1106.0080 [hep-th]]. 
[27] A. Sen, "Logarithmic Corrections to N=2 Black Hole Entropy: An Infrared Window into the Microstates," arXiv:1108.3842 [hep-th]].

[28] S. Ferrara and A. Marrani, "Generalized Mirror Symmetry and Quantum Black Hole Entropy", arXiv:1109.0444 [hep-th]].

[29] D. Shih, A. Strominger and X. Yin, "Counting dyons in N = 8 string theory," JHEP 0606, 037 (2006) arXiv:hep-th/0506151.

[30] A. Sen, "Arithmetic of Quantum Entropy Function," JHEP 0908, 068 (2009) arXiv:0903.1477 [hep-th]].

[31] A. Dabholkar, J. Gomes, S. Murthy, A. Sen, "Supersymmetric Index from Black Hole Entropy," JHEP 1104, 034 (2011). [arXiv:1009.3226 [hep-th]].

[32] R. Dijkgraaf, G. W. Moore, E. P. Verlinde and H. L. Verlinde, "Elliptic genera of symmetric products and second quantized strings," Commun. Math. Phys. 185, 197 (1997) [arXiv:hep-th/9608096].

[33] S. Chaudhuri, G. Hockney and J. D. Lykken, "Maximally supersymmetric string theories in D i 10," Phys. Rev. Lett. 75, 2264 (1995) arXiv:hep-th/9505054].

[34] S. Chaudhuri and J. Polchinski, "Moduli space of CHL strings," Phys. Rev. D 52, 7168 (1995) arXiv:hep-th/9506048].

[35] S. Chaudhuri and D. A. Lowe, "Type IIA heterotic duals with maximal supersymmetry," Nucl. Phys. B 459, 113 (1996) arXiv:hep-th/9508144].

[36] P. S. Aspinwall, "Some relationships between dualities in string theory," Nucl. Phys. Proc. Suppl. 46, 30 (1996) arXiv:hep-th/9508154].

[37] J. Maldacena, G. Moore and A. Strominger, "Counting BPS blackholes in toroidal type II string theory," arXiv:hep-th/9903163.

[38] R. Dijkgraaf, J. M. Maldacena, G. W. Moore, E. P. Verlinde, "A Black hole Farey tail," hep-th/0005003.

[39] S. Carlip, "Logarithmic corrections to black hole entropy from the Cardy formula," Class. Quant. Grav. 17, 4175 (2000) arXiv:gr-qc/0005017.

[40] C. Vafa, "Instantons on D-branes," Nucl. Phys. B463, 435-442 (1996). hep-th/9512078.

[41] J. M. Maldacena, L. Susskind, "D-branes and fat black holes," Nucl. Phys. B475, 679-690 (1996). hep-th/9604042.

[42] S. R. Das, S. D. Mathur, "Excitations of D strings, entropy and duality," Phys. Lett. B375, 103-110 (1996). hep-th/9601152.

[43] S. N. Solodukhin, "The Conical singularity and quantum corrections to entropy of black hole," Phys. Rev. D 51, 609 (1995) [arXiv:hep-th/9407001].

[44] S. N. Solodukhin, "On 'Nongeometric' contribution to the entropy of black hole due to quantum corrections," Phys. Rev. D 51, 618 (1995) [arXiv:hep-th/9408068]. 
[45] D. V. Fursaev, "Temperature And Entropy Of A Quantum Black Hole And Conformal Anomaly," Phys. Rev. D 51, 5352 (1995) arXiv:hep-th/9412161.

[46] R. B. Mann and S. N. Solodukhin, "Conical geometry and quantum entropy of a charged Kerr black hole," Phys. Rev. D 54, 3932 (1996) arXiv:hep-th/9604118.

[47] R. B. Mann and S. N. Solodukhin, "Universality of quantum entropy for extreme black holes," Nucl. Phys. B 523, 293 (1998) [arXiv:hep-th/9709064].

[48] R. K. Kaul and P. Majumdar, "Logarithmic correction to the Bekenstein-Hawking entropy," Phys. Rev. Lett. 84, 5255 (2000) arXiv:gr-qc/0002040].

[49] T. R. Govindarajan, R. K. Kaul and V. Suneeta, "Logarithmic correction to the Bekenstein-Hawking entropy of the BTZ black hole," Class. Quant. Grav. 18, 2877 (2001) arXiv:gr-qc/0104010.

[50] K. S. Gupta, S. Sen, "Further evidence for the conformal structure of a Schwarzschild black hole in an algebraic approach," Phys. Lett. B526, 121-126 (2002). (hep-th/0112041).

[51] A. J. M. Medved, "A comment on black hole entropy or why Nature abhors a logarithm," Class. Quant. Grav. 22, 133 (2005) [arXiv:gr-qc/0406044].

[52] D. N. Page, "Hawking radiation and black hole thermodynamics," New J. Phys. 7, 203 (2005) arXiv:hep-th/0409024.

[53] R. Banerjee and B. R. Majhi, "Quantum Tunneling Beyond Semiclassical Approximation," JHEP 0806, 095 (2008) arXiv:0805.2220 [hep-th]].

[54] R. Banerjee and B. R. Majhi, "Quantum Tunneling, Trace Anomaly and Effective Metric," Phys. Lett. B 674, 218 (2009) arXiv:0808.3688 [hep-th]].

[55] B. R. Majhi, "Fermion Tunneling Beyond Semiclassical Approximation," Phys. Rev. D 79, 044005 (2009) [arXiv:0809.1508 [hep-th]].

[56] R. G. Cai, L. M. Cao and N. Ohta, "Black Holes in Gravity with Conformal Anomaly and Logarithmic Term in Black Hole Entropy," arXiv:0911.4379 [hep-th].

[57] R. Aros, D. E. Diaz and A. Montecinos, "Logarithmic correction to BH entropy as Noether charge," arXiv:1003.1083 [hep-th].

[58] S. N. Solodukhin, "Entanglement entropy of round spheres," Phys. Lett. B693, 605-608 (2010). arXiv:1008.4314 [hep-th]].

[59] S. N. Solodukhin, "Entanglement entropy of black holes," arXiv:1104.3712 [hep-th]].

[60] G. W. Gibbons, S. W. Hawking, "Action Integrals and Partition Functions in Quantum Gravity," Phys. Rev. D15, 2752-2756 (1977).

[61] J. M. Bardeen and G. T. Horowitz, "The extreme Kerr throat geometry: A vacuum analog of AdS(2) x S(2)," Phys. Rev. D 60, 104030 (1999) [arXiv:hep-th/9905099].

[62] D. Astefanesei, K. Goldstein, R. P. Jena, A. Sen and S. P. Trivedi, "Rotating attractors," JHEP 0610, 058 (2006) arXiv:hep-th/0606244. 
[63] H. K. Kunduri, J. Lucietti, H. S. Reall, "Near-horizon symmetries of extremal black holes," Class. Quant. Grav. 24, 4169-4190 (2007). arXiv:0705.4214 [hep-th]].

[64] H. K. Kunduri, J. Lucietti, "A Classification of near-horizon geometries of extremal vacuum black holes," J. Math. Phys. 50, 082502 (2009). [arXiv:0806.2051 [hep-th]].

[65] P. Figueras, H. K. Kunduri, J. Lucietti, M. Rangamani, "Extremal vacuum black holes in higher dimensions," Phys. Rev. D78, 044042 (2008). arXiv:0803.2998 [hep-th]].

[66] A. Sen, "Black hole entropy function and the attractor mechanism in higher derivative gravity," JHEP 0509, 038 (2005) arXiv:hep-th/0506177.

[67] N. Banerjee, S. Banerjee, R. Gupta, I. Mandal and A. Sen, "Supersymmetry, Localization and Quantum Entropy Function," arXiv:0905.2686 [hep-th].

[68] A. Dabholkar, J. Gomes, S. Murthy, "Quantum black holes, localization and the topological string," JHEP 1106, 019 (2011). [arXiv:1012.0265 [hep-th]].

[69] M. J. Duff, "Observations on Conformal Anomalies," Nucl. Phys. B125, 334 (1977).

[70] S. M. Christensen and M. J. Duff, "New Gravitational Index Theorems And Supertheorems," Nucl. Phys. B 154, 301 (1979).

[71] S. M. Christensen and M. J. Duff, "Quantizing Gravity With A Cosmological Constant," Nucl. Phys. B 170, 480 (1980).

[72] M. J. Duff and P. van Nieuwenhuizen, "Quantum Inequivalence Of Different Field Representations," Phys. Lett. B 94, 179 (1980).

[73] S. M. Christensen, M. J. Duff, G. W. Gibbons and M. Rocek, "Vanishing One Loop Beta Function In Gauged N > 4 Supergravity," Phys. Rev. Lett. 45, 161 (1980).

[74] N. D. Birrel and P. C. W. Davis, Quantum Fields in Curved Space, Cambridge University Press, New York, 1982.

[75] P. B. Gilkey, "Invariance theory, the heat equation and the Atiyah-Singer index theorem," Publish or Perish Inc., USA (1984).

[76] D. V. Vassilevich, "Heat kernel expansion: User's manual," Phys. Rept. 388, 279 (2003) [arXiv:hep-th/0306138].

[77] M. J. Duff, S. Ferrara, "Generalized mirror symmetry and trace anomalies," Class. Quant. Grav. 28, 065005 (2011). arXiv:1009.4439 [hep-th]].

[78] R. C. Henry, "Kretschmann scalar for a kerr-newman black hole," astro-ph/9912320.

[79] C. Cherubini, D. Bini, S. Capozziello, R. Ruffini, "Second order scalar invariants of the Riemann tensor: Applications to black hole space-times," Int. J. Mod. Phys. D11, 827-841 (2002). gr-qc/0302095.

[80] A. Castro, J. L. Davis, P. Kraus, F. Larsen, "5D attractors with higher derivatives," JHEP 0704, 091 (2007). hep-th/0702072.

[81] A. Castro, J. L. Davis, P. Kraus, F. Larsen, "5D Black Holes and Strings with Higher Derivatives," JHEP 0706, 007 (2007). hep-th/0703087]. 
[82] A. Castro, J. L. Davis, P. Kraus, F. Larsen, "Precision Entropy of Spinning Black Holes," JHEP 0709, 003 (2007). arXiv:0705.1847 [hep-th]].

[83] A. Castro, J. L. Davis, P. Kraus, F. Larsen, "String Theory Effects on Five-Dimensional Black Hole Physics," Int. J. Mod. Phys. A23, 613-691 (2008). arXiv:0801.1863 [hep-th]].

[84] A. Castro and S. Murthy, "Corrections to the statistical entropy of five dimensional black holes," JHEP 0906, 024 (2009) arXiv:0807.0237 [hep-th]].

[85] B. de Wit, S. Katmadas, "Near-horizon analysis of D=5 BPS black holes and rings," JHEP 1002, 056 (2010). arXiv:0910.4907 [hep-th]].

[86] P. D. Prester, " $A d S_{3}$ backgrounds from 10D effective action of heterotic string theory," arXiv:0912.0030 [hep-th].

[87] P. D. Prester, "alpha'-corrections and heterotic black holes," arXiv:1001.1452 [hep-th].

[88] M. Cvitan, P. D. Prester and A. Ficnar, "alpha ${ }^{2}$-corrections to extremal dyonic black holes in heterotic string theory," JHEP 0805, 063 (2008) [arXiv:0710.3886 [hep-th]].

[89] P. D. Prester and T. Terzic, "alpha'-exact entropies for BPS and non-BPS extremal dyonic black holes in heterotic string theory from ten-dimensional supersymmetry," JHEP 0812, 088 (2008) arXiv:0809.4954 [hep-th]].

[90] A. Dabholkar, A. Sen and S. P. Trivedi, "Black hole microstates and attractor without supersymmetry," JHEP 0701, 096 (2007) arXiv:hep-th/0611143.

[91] N. Banerjee, I. Mandal, A. Sen, "Black Hole Hair Removal," JHEP 0907, 091 (2009). arXiv:0901.0359 [hep-th]].

[92] S. Cecotti, P. Fendley, K. A. Intriligator, C. Vafa, "A New supersymmetric index," Nucl. Phys. B386, 405-452 (1992). hep-th/9204102.

[93] C. Bachas and E. Kiritsis, "F**4 terms in $\mathrm{N}=4$ string vacua," Nucl. Phys. Proc. Suppl. 55B, 194 (1997) arXiv:hep-th/9611205.

[94] A. Gregori, E. Kiritsis, C. Kounnas, N. A. Obers, P. M. Petropoulos and B. Pioline, "R**2 corrections and non-perturbative dualities of $\mathrm{N}=4$ string ground states," Nucl. Phys. B 510, 423 (1998) arXiv:hep-th/9708062.

[95] D. Gaiotto, A. Strominger, X. Yin, "New connections between 4-D and 5-D black holes," JHEP 0602, 024 (2006). hep-th/0503217].

[96] N. Banerjee, "Subleading Correction to Statistical Entropy for BMPV Black Hole," Phys. Rev. D 79, 081501 (2009) [arXiv:0807.1314 [hep-th]].

[97] A. Sen, "Black Hole Entropy Function, Attractors and Precision Counting of Microstates," Gen. Rel. Grav. 40, 2249 (2008) arXiv:0708.1270 [hep-th]].

[98] D. P. Jatkar, A. Sen, Y. K. Srivastava, "Black Hole Hair Removal: Non-linear Analysis," JHEP 1002, 038 (2010). [arXiv:0907.0593 [hep-th]].

[99] R. Camporesi and A. Higuchi, "Spectral functions and zeta functions in hyperbolic spaces," J. Math. Phys. 35, 4217 (1994). 
[100] R. Camporesi and A. Higuchi, "On The Eigen Functions Of The Dirac Operator On Spheres And Real Hyperbolic Spaces," J. Geom. Phys. 20, 1 (1996) arXiv:gr-qc/9505009.

[101] C. Beasley, D. Gaiotto, M. Guica, L. Huang, A. Strominger and X. Yin, "Why Z(BH) = -Z(top) —**2," arXiv:hep-th/0608021. 